\title{
Processing and Thermoelectric Properties of TiNiSn Materials: A Review
}

\author{
J.S. Young and R.G. Reddy
}

(Submitted July 2, 2019; in revised form September 23, 2019; published online October 15, 2019)

\begin{abstract}
The half-Heusler alloy TiNiSn is a promising material for high-temperature thermoelectric applications due to its good thermal stability and semiconductor-like electrical properties. Numerous synthesis techniques have been utilized to make TiNiSn, but a comparative study on its thermoelectric properties with respect to processing parameters has not been reported. Based on published experimental data, primary melting methods, such as arc melting or induction levitation melting, do not have a noticeable effect on the final thermoelectric properties, although choice of densification technique and annealing parameters correlate with each thermoelectric property. Thermal conductivity $(2.47-6.08 \mathrm{~W} / \mathrm{m} \mathrm{K})$, governed by lattice scattering effects, was maximized with high density from the spark plasma sintering (SPS) technique as well with low inclusions of metallic impurities and interstitial Ni defects from low-temperature, non-densified processes. Electrical resistivity $(4.75-30 \mu \Omega \mathrm{m})$, inversely related to thermal conductivity, is minimized when density is maximized and chemical defects are favorable from using SPS. All reported TiNiSn alloys contain intermetallic impurities, imparting a global trend of lowered electrical resistivity. Seebeck coefficient $(-273.20$ to $-\mathbf{5 0 . 7 1} \mu \mathrm{V} / \mathrm{K}$ ), proportional to electrical resistivity, is maximized when charge carrier concentration is minimized by eliminating phase impurities and Ni defects. Porosity has been observed to scatter low-energy electrons, which can increase Seebeck coefficient and electrical resistivity, although no statistical significance is found. An ideal TiNiSn alloy should have intermediate values of all interconnected thermoelectric properties to maximize $Z T$. Future research work should strive to include quantitative phase analysis to better characterize the contributions of $\mathrm{Ni}$ defects and impurity phases toward thermoelectric properties.
\end{abstract}

Keywords electrical resistivity, half-Heusler, heat treatment, intermetallic, Seebeck coefficient, thermal conductivity, thermoelectric

\section{Introduction}

A proposed solution to meet the demand for more efficient energy generation and reduced fuel consumption is through the use of thermoelectric materials. Utilizing the Seebeck and Peltier effects, thermoelectric materials have the unique property to convert waste heat into useful electrical energy and vice versa (Ref 1). Primary power generation applications can require temperature differentials of $800 \mathrm{~K}$ or higher (Ref 2). Thermoelectric material efficiency is judged on their figure of merit (ZT), a dimensionless property, expressed as:

$Z T=\frac{S^{2} T}{\rho k_{\mathrm{tot}}}$

where $S$ is the Seebeck coefficient, $\rho$ is the electrical resistivity, $k_{\text {tot }}$ is the total thermal conductivity, and $T$ is the applied temperature. A high $Z T$ value indicates an increased energy conversion efficiency.

J.S. Young and R.G. Reddy, Department of Metallurgical and Materials Engineering, The University of Alabama, Tuscaloosa, AL 35487. Contact e-mails: jsyoung4@crimson.ua.edu and rreddy@eng.ua.edu.
Many classes of materials exhibit a thermoelectric effect, where a small energy bandgap promotes a high carrier concentration. Since thermoelectric efficiency is a function of operational temperature (Eq 1), material selection is a critical decision to maximize $Z T$ for a specific application, summarized recently by Bogala (Ref 3), Dehkordi et al. (Ref 4), Hébert et al. (Ref 5), and Chen and Ren (Ref 6). Low-temperature applications $(25-600 \mathrm{~K})$ utilize highly efficient tellurides (Ref 7) and skutterudites (Ref 8) that are not thermally stable near 600 K. Metal silicides (Ref 9, 10) and half-Heuslers (Ref 11, 12) are optimal at high temperatures $(600-800 \mathrm{~K})$, while metal oxides (Ref 13), germanides (Ref 14), and antimonides (Ref 15) are preferred for extreme temperature applications $(>800 \mathrm{~K})$.

Half-Heuslers, a promising group of thermoelectric compounds, are a class of intermetallic alloys that show many similarities to semiconductors due to their bandgap around the Fermi level, providing a lower thermal conductivity and large Seebeck coefficient at elevated temperatures (Ref 16). Compared to other thermoelectric material classes, doped halfHeusler alloys provide optimal conversion efficiency at intermediate temperatures and are generally composed of low-cost elements abundant in nature. The natural vacancies within the half-Heusler unit cell favor easy doping of either impurity or parent elements, strengthening their electronic properties. The half-Heusler alloy TiNiSn has been systematically investigated by many scientists based on its excellent thermal stability, nontoxicity, high $Z T$ at elevated temperatures, and ease of doping through vacancy or substitution defects (Ref 16-18).

Synthesis of pure TiNiSn can prove difficult due to the complexity of the TiNiSn phase diagram and the low melting point of Sn (Ref 19). The TiNiSn phase possesses a small phase stability window while $\mathrm{TiNi}_{2} \mathrm{Sn}$ and other multi-element phases 
are comparatively large. This aspect hinders the synthesis of pure TiNiSn due to the prevalent formation of localized regions of non-homogeneity. Various processing techniques of TiNiSn have been investigated to enhance purity, density, and ultimately ZT, but a complete, comparative analysis of these methods is not available in the literature.

This work is a comprehensive review of the conventional synthesis and processing parameters of pure TiNiSn utilized thus far in the literature, as well as an analysis on how processing technique affects $Z T$ and its contributing thermoelectric properties. A focus will be made on each thermoelectric property at elevated temperatures $(\sim 600-800 \mathrm{~K})$. Through this analysis, some inference on optimal processing parameters can be made in order to maximize $Z T$ for future TiNiSn-based research as well as on other half-Heusler compounds. Additionally, gaps in current knowledge can be identified in order to more accurately correlate property/processing relationships going forth.

\section{Processing Parameter Overview}

\subsection{Arc Melting}

Vacuum arc melting (AM) is a prevalent technique for synthesis of bulk metal alloys. In a laboratory setting, a TIG welding unit provides power to an electrode, commonly tungsten, where an arc is struck onto a sample on a watercooled copper hearth. A large amount of heat is concentrated onto the sample from the electric arc, melting the metal into an alloy ingot. Cycles of melting and flipping of the sample improve phase homogeneity, but localized regions of nonhomogeneity are common.

Arc melting is often utilized to consolidate powders into a bulk sample while providing enough heat energy to form stable phases (Ref 20-22). While the arc melting technique is useful due to a quick experimental duration and low monetary cost, numerous limitations exist which require additional techniques to address. All examples of arc-melted TiNiSn employ secondary processing techniques afterward since pure TiNiSn is not formed from arc melting alone due to a small phase region and slow diffusion kinetics (Ref 19, 23, 24). Additionally, sample temperature and heating/cooling rate are only indirectly controlled via applied arc current. Melt compositions with solidification paths that involve phases with invariant reactions (e.g., $\delta$ in $\mathrm{Cu}-\mathrm{Zn}$ phase diagram (Ref 25)) can promote unwanted phases with slow heating and cooling rates. Impurity metallic phases, such as $\mathrm{TiNi}_{2} \mathrm{Sn}$ and $\mathrm{Ti}_{5} \mathrm{Sn}_{3}$, can result in decreases in Seebeck coefficient and electrical resistivity (Ref 23).

\subsection{Induction Levitation Melting}

The levitation melting (LM) technique can melt high melting point alloys without contamination from a crucible material. The sample is levitated using electromagnetic forces generated by a coil, while being simultaneously molten and inductively mixed (Ref 26, 27). Benefits of levitation melting include prevention of impurity elements, a negligible temperature gradient throughout the sample, and thorough mixing to ensure phase homogeneity. Disadvantages of induction levitation melting come from instrument limitations and use of electromagnetics. Sample size melted at one time is limited up to $1 \mathrm{~kg}$ due to droplet stability difficulties (Ref 28). Additionally, since levitation and heat produced are coupled together to the electromagnetic coil, material selection and size need to be carefully considered in order to prevent vaporization of low vapor pressure elements (Ref 29). Finally, the density of induction-melted samples is lower due to residual porosity content from the lack of applied pressure during processing, which will affect mechanical strength and phonon transport (Ref 30, 31).

\subsection{Microwave Synthesis}

Solid-state reactions using microwaves (MW) have been used since the 1970s to synthesize metallic, ceramic, and thermoelectric materials (Ref 32). Materials can couple with the microwave radiation to enable uniform heating throughout the sample, a property termed as the material dielectric loss tangent. Susceptor materials can be used to aid heating if a sample's dielectric loss tangent is naturally low. This dielectric heating promotes rapid atomic diffusion between reactants to achieve the desired phase composition in a short duration, negating the need for secondary thermal treatments (Ref 33). Grain growth is inhibited since sintering duration is short, promoting phonon scattering due to higher grain boundary density. With extended microwave exposure $(\sim 0.5 \mathrm{~h})$, the sample powders can also be sintered into a bulk piece (Ref 34$)$. The primary complication of microwave sintering is the low final density of the bulk sample, where numerous nanopores are produced and can have a negative effect on charge carrier mobility (Ref 34, 35). Additionally, the cold compaction of powder before microwave synthesis can have trapped oxygen, exhibited in trace amounts of oxide phase in the final product (Ref 34).

\subsection{Annealing}

Annealing (AN) is a secondary heat treatment process which is used to alter the physical and chemical properties of a material to their equilibrium states through the application of heat while staying below the melting point (Ref 20, 36). Annealing is separated into three stages: recovery, recrystallization, and grain growth, information of which can be found readily in the literature (Ref 37 ). Most synthesis techniques of TiNiSn produce small quantities of impurity phases, such as Sn, $\mathrm{Ti}_{6} \mathrm{Sn}_{5}$, and $\mathrm{TiNi}_{2} \mathrm{Sn}$, due to the crystallization path and narrow phase field of TiNiSn (Ref 24). Annealing TiNiSn for long durations ( $>1$ week) is a standard procedure to reduce impurity content and produce an equilibrium phase composition. While the phase purity is improved, long heat treatments promote grain growth, reducing grain boundary density and increasing thermal conductivity, a detriment to $Z T$ (Eq 1).

\subsection{Spark Plasma Sintering}

Spark plasma sintering (SPS), more accurately described as pulsed electrical current sintering, is a powder consolidation technique for metals and ceramics where high pressure and a pulsed DC current are applied simultaneously. High heating rates up to $1000 \mathrm{~K} / \mathrm{s}$ can be achieved, enabling near-complete densification of powders and low grain growth (Ref 38). Production of a small amount of plasma can generate a cleansing effect, removing adsorbed material from the surface, such as $\mathrm{CO}_{2}$ and $\mathrm{H}_{2} \mathrm{O}$, ultimately aiding densification (Ref 39). SPS has been used as a significantly shorter duration alternative 
( $<10 \mathrm{~min}$ ) to melting and annealing in order to achieve a similarly high phase purity TiNiSn. Limitations of SPS are primarily commercial in nature. Samples are currently limited to small symmetrical shapes (e.g., cylinder), which may not be optimal for thermoelectric devices. Since SPS uses DC electrical current to generate heat, adequate electrical conductivity of starting powders is critical to achieve a low thermal gradient and homogenous sintering behavior (Ref 40).

\subsection{Hot Isostatic Pressing}

Traditional sintering is the incomplete fusion of particles during which a powder compact is exposed to an elevated temperature. Hot isostatic pressing (HIP) is the sintering of powders at high temperature with an applied isostatic pressure (Ref 41). This combination of heat and pressure enhances the driving forces for diffusion and many sintering mechanisms, such as capillary forces, rearrangement of particles, surface tension, and induced creep and plasticity in the powder sample, enabling compaction of traditionally difficult to manufacture and expensive materials (Ref 42). HIP is considered an alternative to SPS and microwave sintering (MWS), although with a longer duration ( $\geq 1 \mathrm{~h}$ ) than both due to a reliance on slow kinetics of heat transfer from the container to powders, final microstructures can have comparatively larger grain sizes (Ref 40, 43, 44). While SPS is limited to rigid dies and simple geometries, HIP allows for many complex geometries enabled by uniform shrinkage due to the isostatic pressure ( $\operatorname{Ref} 45,46)$.

\subsection{Reaction Sintering}

Reaction sintering (RS), more commonly known as liquid phase sintering, is an accelerated sintering variant where a precursor liquid surrounds the solid grains, enhancing densification and diffusion rates due to capillary forces and large atom mobility, in order to produce a dense phase or composite (Ref 47). Kimura et al. (Ref 48) produced nearly pure TiNiSn through RS and AN of TiNi and Sn precursor powders. Since $\mathrm{Sn}$ has a low melting point compared to $\mathrm{Ti}$ and $\mathrm{Ni}$, it melts during sintering and diffuses rapidly into the TiNi bulk structure, forming TiNiSn at the interfaces. Unique layered microstructures can be produced via reaction sintering, where a fine-grained layer of TiNiSn is formed toward the TiNi side, while coarse TiNiSn facets are formed into the Sn phase (Ref 48). A non-homogenous precursor material means varying solidification paths toward the TiNiSn phase region, producing common metallic impurity phases that require secondary heat treatment (e.g., annealing) to eliminate. Additionally, lack of applied pressure during sintering forms a porous microstructure and charge carrier scattering, demanding SPS or HIP after reaction sintering.

\subsection{Reported Synthesis Parameters}

The previously mentioned synthesis techniques have been utilized in combination with one another for TiNiSn alloys, as well as bulk thermoelectric materials in general, for over a decade. Despite an abundance of experimental data, a thorough comparison of processing techniques has yet to be completed. Table 1 summarizes the unique procedures reported and relevant experimental parameters for synthesizing pure TiNiSn for thermoelectric applications.

Regardless of reported method from Table 1, various impurities within the TiNiSn matrix exist in each final product, as summarized in Table 2. Figure 1 presents a TiNiSn alloy microstructure with impurity phases present after three weeks of annealing. The metallic full-Heusler phase, $\mathrm{TiNi}_{2} \mathrm{Sn}$, is the most commonly observed impurity. Impurity phases reported in the literature are mostly noted qualitatively by XRD and microscopy. It can be assumed the TiNiSn matrix purity for non-densified methods to be about $97-98 \%$ based on Lei et al. (Ref 34), Douglas et al. (Ref 31), and Young (Ref 24). The impurity phase percentages for methods involving HIP or SPS are not reported.

\section{Processing Parameter Effects}

\subsection{Thermal Conductivity}

Applied temperature gradients to a solid material produce a heat flux, where the ability of the material to transfer heat is its thermal conductivity. The heat flux in semiconductors is caused by the transmission of energy through both phonons and free electrons to less excited, lower temperature regions. For thermoelectrics, maximizing $Z T$ involves low thermal conductivity and large electrical conductivity, so carrier concentration tuning is necessary. Additionally, thermal conductivity can be lowered through phonon scattering, a product of the reduction in the mean-free path of electrons through the introduction of crystal defects (point defects, dislocations, impurities, and grain boundaries) (Ref 37). Figure 2 shows a wide range of total thermal conductivities at elevated temperatures of TiNiSn as synthesis process is changed.

Overall, total thermal conductivity of TiNiSn, ranging from 2.47 to $6.08 \mathrm{~W} / \mathrm{m} \mathrm{K}$, has a convex parabolic trend with increasing temperature, where the minimum is found at elevated temperatures. Ding et al. (Ref 49) calculated the theoretical thermal conductivity of TiNiSn (dashed line) using first principles, finding that it was controlled by lattice contributions.

Density shows a clear relationship with synthesis technique, where the theoretical density of TiNiSn is $7.21 \mathrm{~g} / \mathrm{cm}^{3}$. All processes utilizing SPS create $>99 \%$ dense samples, while processes using other densification techniques, such as HIP or MWS, or using none at all report more porous microstructures below $7 \mathrm{~g} / \mathrm{cm}^{3}$, compared in Fig. 3. Density also experimentally correlates with $\sim 94 \%$ certainty $(p=0.062)$ to thermal conductivity, where processes including SPS have a larger density and also high conductivity, as illustrated in Fig. 4. This can be attributed to the phonon scattering effect of less dense materials that contain point defects and bulk perturbations of the crystal (i.e., pores, cracks), which reduces the mean-free path of phonons and ultimately reduces thermal conductivity (Ref 37).

Sources of uncertainty related to sample density can be attributed to the impurity phases present in the TiNiSn matrix. Since the volume percentages and equilibrium phase contents vary between processes (Table 2), theoretical 100\% density deviates from pure TiNiSn. Densities are commonly reported only qualitatively or as a percentage, which leaves the reference density unclear. The estimated densities in Table 3 are calculated based on the pure TiNiSn theoretical density.

Grain size and grain boundary morphology can also play a role toward phonon scattering, where a smaller grain size generates more grain boundaries (crystal defect) and can lower 


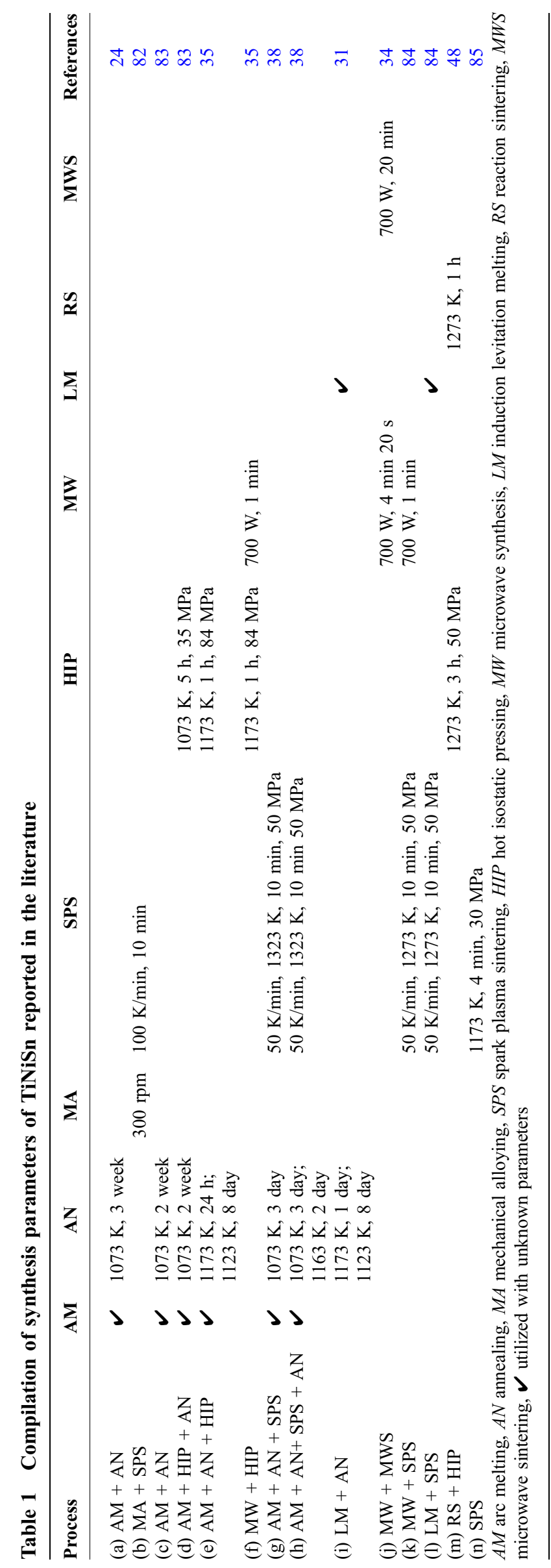


thermal conductivity. Schrade et al. (Ref 50) theoretically and experimentally found that nano-sized grains were an effective method of lowering thermal conductivity and its dependence on temperature in half-Heusler materials. Bhattacharya et al. (Ref 51) found a somewhat linear relationship between lattice thermal conductivity and grain size diameter in $\mathrm{TiNiSn}_{1-x} \mathrm{Sb}_{x}$. Additionally, Douglas et al. (Ref 31 ) proposed that increased amounts of secondary phases, such as $\mathrm{TiNi}_{2} \mathrm{Sn}$, reduce lattice thermal conductivity due to interfacial scattering in $\mathrm{TiNi}_{1+x} \mathrm{Sn}$.

Table 2 Reported impurity phases in TiNiSn alloy by $\mathrm{x}$-ray diffraction and microscopy

\begin{tabular}{ll}
\hline Process & $\mathrm{Reported} \mathrm{impurity} \mathrm{phases}$ \\
\hline (a) $\mathrm{AM}+\mathrm{AN}$ & $\mathrm{TiNi}_{2} \mathrm{Sn}, \mathrm{Ni}_{3} \mathrm{Sn}_{4}$ \\
(b) $\mathrm{MA}+\mathrm{SPS}$ & $\mathrm{TiNi}_{2} \mathrm{Sn}, \mathrm{Ni}_{3.39} \mathrm{Sn}_{4}, \mathrm{TiO}_{2}$ \\
(c) $\mathrm{AM}+\mathrm{AN}$ & $\mathrm{Ti}_{6} \mathrm{Sn}_{5}, \mathrm{Sn}$ \\
(d) $\mathrm{AM}+\mathrm{HIP}+\mathrm{AN}$ & $\mathrm{Ti}_{6} \mathrm{Sn}_{5}$ \\
(e) $\mathrm{AM}+\mathrm{AN}+\mathrm{HIP}$ & $\mathrm{TiNi}_{2} \mathrm{Sn}$ \\
(f) $\mathrm{MW}+\mathrm{HIP}$ & $\mathrm{TiNi}_{2} \mathrm{Sn}, \mathrm{Ti}_{5} \mathrm{Sn}_{3}$ \\
(g) $\mathrm{AM}+\mathrm{AN}+\mathrm{SPS}$ & $\mathrm{TiNi}_{2} \mathrm{Sn}, \mathrm{Ti}_{6} \mathrm{Sn}_{5}, \mathrm{Sn}$ \\
(h) $\mathrm{AM}+\mathrm{AN}+\mathrm{SPS}+\mathrm{AN}$ & $\mathrm{TiNi}_{2} \mathrm{Sn}, \mathrm{Sn}$ \\
(i) $\mathrm{LM}+\mathrm{AN}$ & $\mathrm{TiNi}_{2} \mathrm{Sn}, \mathrm{Ti}_{6} \mathrm{Sn}_{5}, \mathrm{Ni}_{3} \mathrm{Sn}_{4}$ \\
(j) $\mathrm{MW}+\mathrm{MWS}$ & $\mathrm{TiNi}_{2} \mathrm{Sn}, \mathrm{Ti}_{2} \mathrm{Sn}_{2} \mathrm{Ti}_{1} \mathrm{Sn}$ \\
(k) $\mathrm{MW}+\mathrm{SPS}$ & $\mathrm{TiNi}_{2} \mathrm{Sn}, \mathrm{Ti}_{6} \mathrm{Sn}_{5}$ \\
(l) $\mathrm{LM}+\mathrm{SPS}$ & $\mathrm{TiNi}_{2} \mathrm{Sn}, \mathrm{Ti}_{6} \mathrm{Sn}_{5}, \mathrm{Ni}_{3} \mathrm{Sn}_{4}, \mathrm{Sn}$ \\
(m) $\mathrm{RS}+\mathrm{HIP}$ & $\mathrm{TiNi}_{2} \mathrm{Sn}$ \\
(n) $\mathrm{SPS}$ & $\mathrm{TiNi}_{2} \mathrm{Sn}, \mathrm{Sn}$ \\
\hline
\end{tabular}

This was correlated with a smaller difference in thermal conductivities between $\mathrm{TiNiSn}$ and $\mathrm{TiNi}_{1.15} \mathrm{Sn}$ as temperature increased, due to a larger influence of electronic thermal conductivity from $\mathrm{TiNi}_{2} \mathrm{Sn}$. Larger intermetallic $\mathrm{TiNi}_{2} \mathrm{Sn}$ content also increases charge carrier concentration, which can increase electronic thermal conductivity. While experimental grain size ranges from 0.05 to $76 \mu \mathrm{m}$ in Table 3 , there is not enough quantitative data on TiNiSn morphology to confirm that it is significantly correlated with thermal conductivity using ANOVA $(p=0.267)$.

\subsection{Electrical Resistivity}

Electrical conductance is a function of a material's band structure, where the distance between its valence and conduction bands is the energy bandgap, $E_{\mathrm{g}}$. Promotion of electrons from the valence to the conduction band for free movement through a material requires an energy input equal to the bandgap. A large bandgap induces a smaller charge carrier concentration and higher electrical resistivity. TiNiSn, an intrinsic semiconducting material, has a theoretical bandgap between 0.40 (Ref 52) and $0.51 \mathrm{eV}$ (Ref 53). Bandgap can be estimated using an Arrhenius relationship by:

$\ln (\sigma)=\ln \left(\sigma_{0}\right)+\left(\frac{-E_{\mathrm{g}}}{2 k_{\mathrm{B}}}\right)\left(\frac{1}{T}\right)$

where $\sigma$ is the electrical conductivity, $E_{\mathrm{g}}$ is the activation energy required for electron promotion (i.e., bandgap), and $k_{\mathrm{B}}$
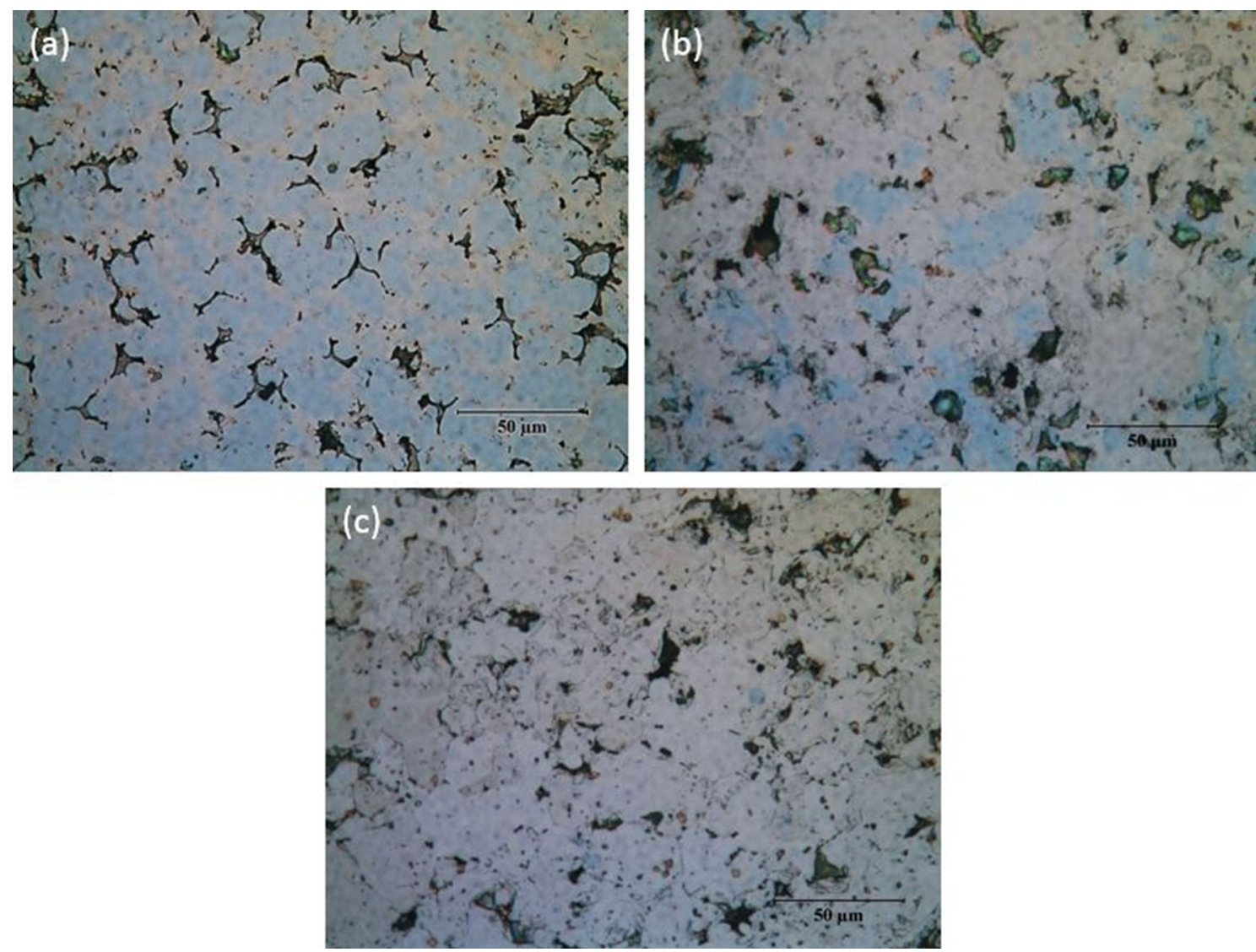

Fig. 1 Optical microstructure of TiNiSn alloy, etched with Kroll's reagent; arc-melted and annealed at $1073 \mathrm{~K}$ for (a) 0 weeks/as-cast, (b) 1 week, and (c) 3 weeks. White regions are TiNiSn, blue is $\mathrm{TiNi}_{2} \mathrm{Sn}$, orange is $\mathrm{Ti}$, and black is porosity (Ref 24). Images reproduced with permission (Color figure online) 
is the Boltzmann constant. The change in electrical resistivity of TiNiSn with synthesis parameter is shown in Fig. 5. Theoretical bandgap is calculated (Eq 2) and listed in Table 4.

TiNiSn follows general semiconductor behavior, as shown in Fig. 5, whereas temperature increases and electrical resistivity decreases due to increased charge carrier density.

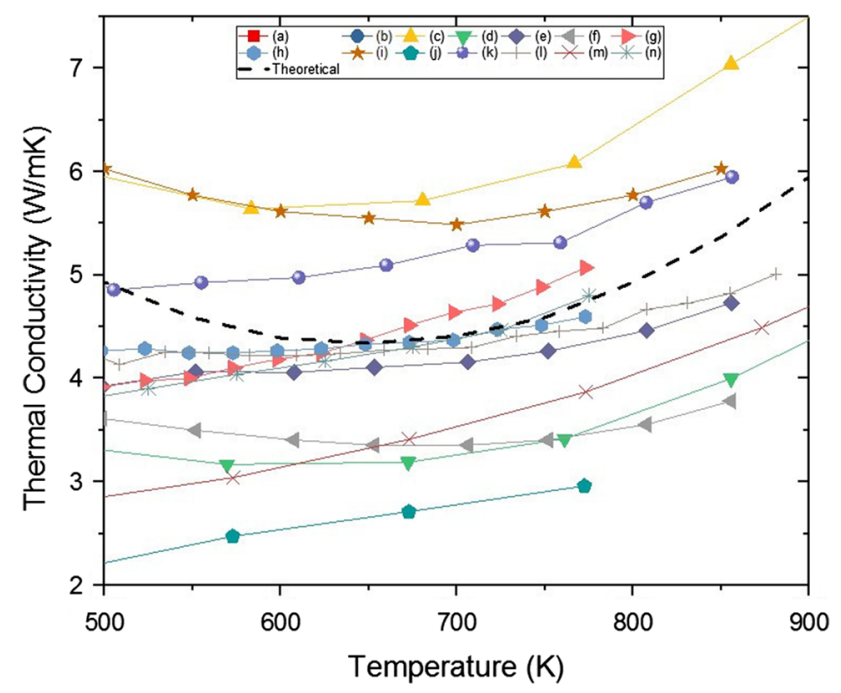

Fig. 2 Total thermal conductivity (W/m K) vs. temperature (K) for selected synthesis parameters of TiNiSn, extracted from the literature
Electrons are more freely able to jump to the conduction band, and consequently, holes are generated and flow in the valence band. Berche and Jund (Ref 54) calculated theoretical electrical resistivity of TiNiSn (dashed line), based on a bandgap of $0.12 \mathrm{eV}$.

In terms of $Z T$, electrical resistivity should be minimized. While processes $a$ and $b$ have the lowest resistivity, both exhibit a metallic behavior where resistivity increases with temperature and their bandgap is $<0 \mathrm{eV}$. A positive, nonzero bandgap is necessary for reasonable Seebeck coefficients and ZT, expanded upon in subsequent sections.

Considering processes $a$ and $b$ as outliers, regardless of melting parameters, samples densified with SPS have a lower resistivity than samples using HIP or no densification process using a Tukey test $(p<0.05)$. This can be attributed to the density (i.e., porosity), as listed in Table 2. Downie et al. (Ref 55) found that reduced porosity led to a substantial decrease in resistivity for $\mathrm{Ti}_{1-x} \mathrm{Zr}_{x} \mathrm{NiSn}$. Porosity increases resistivity by increasing the mean path length for electrical flow to pass (Ref 56). This relationship is illustrated in Fig. 6, where statistical significance between density and electrical resistivity is confirmed $(p=0.042)$.

Resistivity can also be heightened through induced defects, such as grain boundaries, dislocations, and point defects, by increasing the scattering of conducting electrons (Ref 57). Hazama et al. (Ref 58) found that non-stoichiometry, such as excess nickel, formed $\mathrm{TiNi}_{1+x} \mathrm{Sn}$, where $\mathrm{Ni}$ atoms filled naturally vacant interstitial sites to induce defects in the TiNiSn
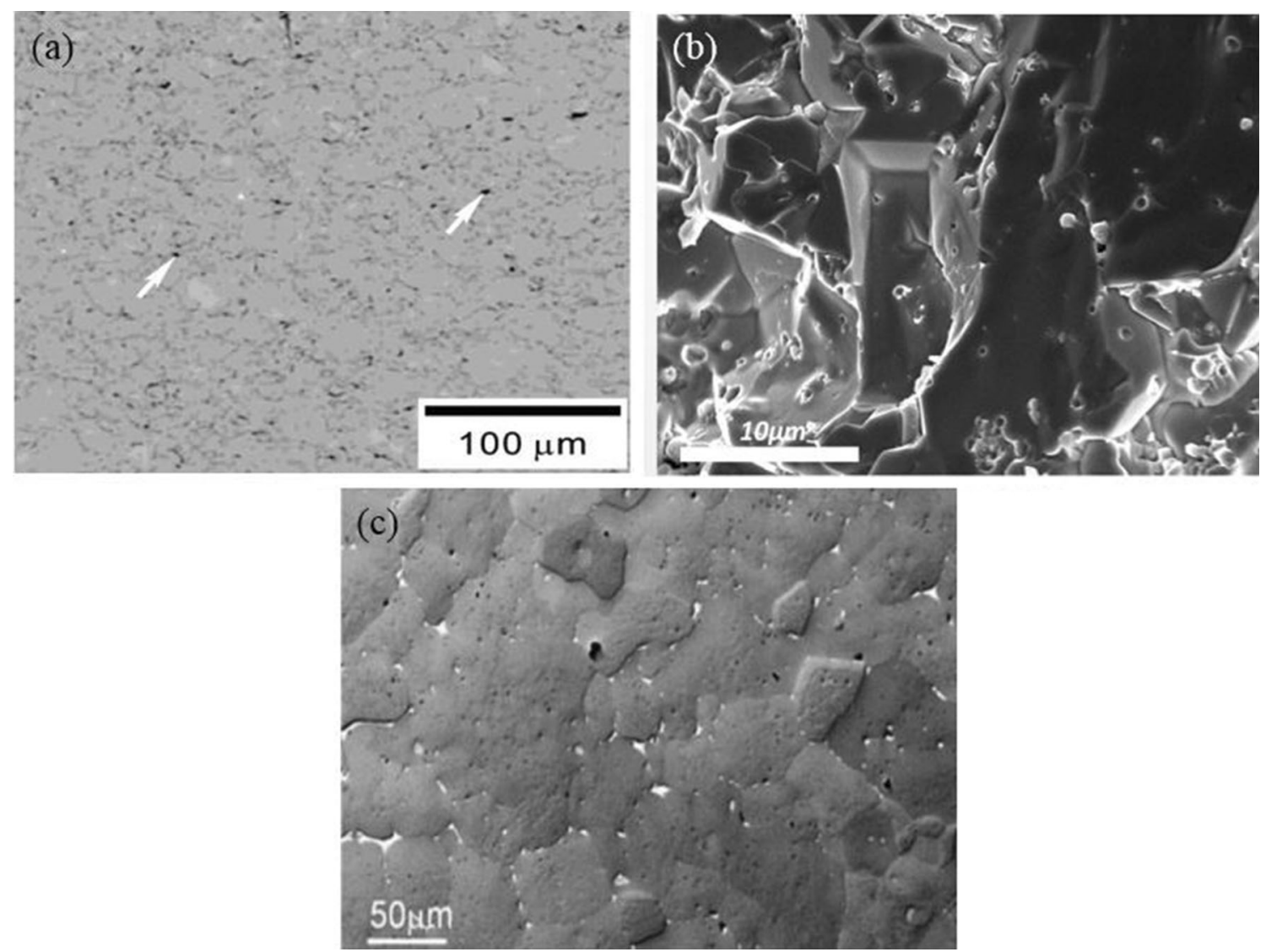

Fig. 3 (a) SEM micrograph of TiNiSn synthesized by AM, RS, and HIP (Ref 87); (b) TEM image of TiNiSn bulk alloy with nanopores synthesized via MWS (Ref 34); (c) back scattered SEM micrograph of TiNiSn after $72 \mathrm{~h}$ heat treatment synthesized using SPS (Ref 38). All images reproduced with permission 


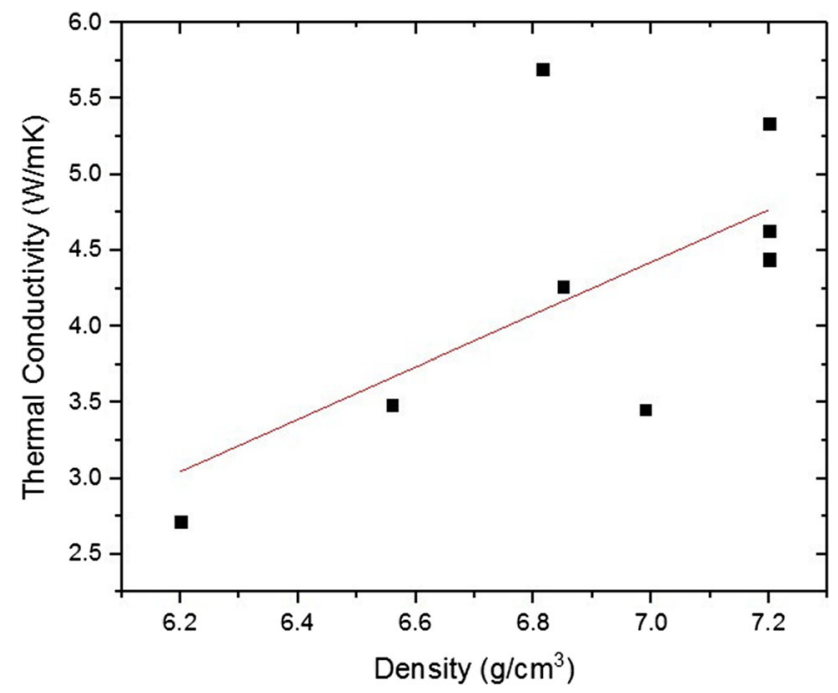

Table 4 Calculated bandgap of TiNiSn with reported synthesis process

Synthesis process

Bandgap, eV

(b) MA + SPS

$-0.005$

(a) $\mathrm{AM}+\mathrm{AN}$

$-0.007$

(g) $\mathrm{AM}+\mathrm{AN}+\mathrm{SPS}$

0.094

(n) SPS

0.021

(k) MW + SPS

0.083

(l) $\mathrm{LM}+\mathrm{SPS}$

0.111

(m) RS + HIP

0.129

(h) $\mathrm{AM}+\mathrm{AN}+\mathrm{SPS}+\mathrm{AN}$

0.106

(j) $\mathrm{MW}+\mathrm{MWS}$

0.079

(d) $\mathrm{AM}+\mathrm{HIP}+\mathrm{AN}$

0.155

(i) $\mathrm{LM}+\mathrm{AN}$

0.140

(f) $\mathrm{MW}+\mathrm{HIP}$

0.101

(e) $\mathrm{AM}+\mathrm{AN}+\mathrm{HIP}$

0.072

(c) $\mathrm{AM}+\mathrm{AN}$

0.197

Fig. 4 Average thermal conductivity (W/m K) vs. density $\left(\mathrm{g} / \mathrm{cm}^{3}\right)$ of selected synthesis parameters of TiNiSn from 600 to $800 \mathrm{~K}$

Table 3 Density and average grain size of TiNiSn with reported synthesis process

Synthesis process Estimated density, $\mathbf{g} / \mathrm{cm}^{3}$

Grain size, $\mu \mathrm{m}$

(j) MW + MWS

6.2

0.1

(d) $\mathrm{AM}+\mathrm{HIP}+\mathrm{AN}$

...

(f) $\mathrm{MW}+\mathrm{HIP}$

6.56

6.99

(m) RS + HIP

6.85

$1-0.13$

(e) $\mathrm{AM}+\mathrm{AN}+\mathrm{HIP}$

7.2

(n) SPS

(h) $\mathrm{AM}+\mathrm{AN}+\mathrm{SPS}+\mathrm{AN}$

...

5-10

$0.11-0.13$

(g) $\mathrm{AM}+\mathrm{AN}+\mathrm{SPS}$

7.2

(k) $\mathrm{MW}+\mathrm{SPS}$

7.2

...

(i) $\mathrm{LM}+\mathrm{AN}$

7.2

$6.71-6.92$

0.05

76

45

...

(c) $\mathrm{AM}+\mathrm{AN}$

...

$20-30$

Theoretical density of TiNiSn is $7.21 \mathrm{~g} / \mathrm{cm}^{3}(\operatorname{Ref} 86)$

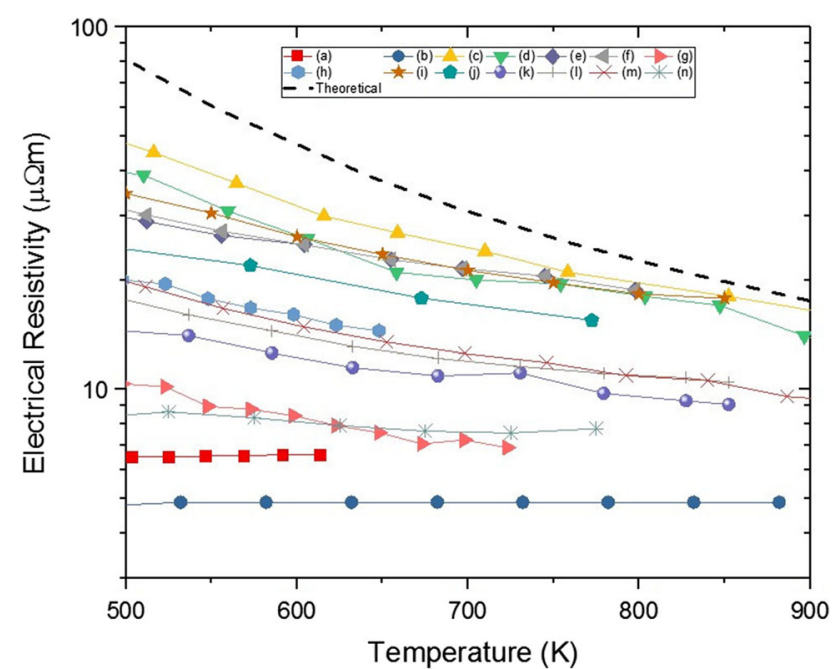

Fig. 5 Electrical resistivity ( $\mu \Omega \mathrm{m})$ vs. temperature $(\mathrm{K})$ for selected synthesis parameters of TiNiSn, extracted from the literature

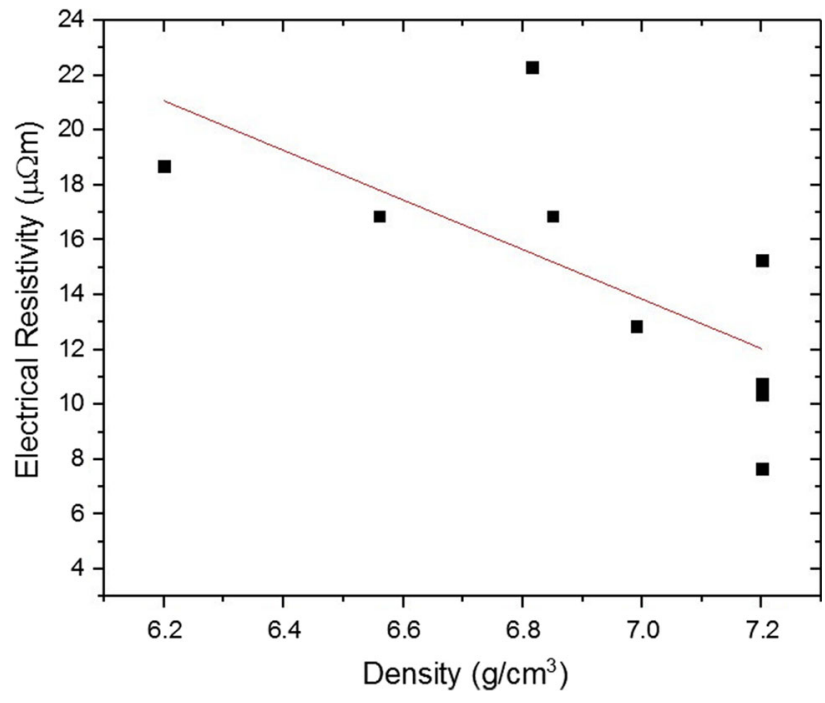

Fig. 6 Average electrical resistivity $(\mu \Omega \mathrm{m})$ vs. density $\left(\mathrm{g} / \mathrm{cm}^{3}\right)$ of selected synthesis parameters of TiNiSn from 600 to $800 \mathrm{~K}$ 
unit cell, randomly distributed throughout the entire bulk phase, as shown in Fig. 7. Tang et al. (Ref 59) further found that from $0 \leq X_{\mathrm{Ni}} \leq 0.06$, as temperature and $\mathrm{Ni}$ content increased (experimentally up to $1223 \mathrm{~K}$ ), the stable phase region of TiNiSn widened, ultimately allowing more stable Ni interstitialfilled sites. Downie et al. (Ref 55) suggest that SPS does introduce some interstitial Ni based on their diffraction data and fitted activation energies, resulting in additional in-gap states and a reduced bandgap, although this effect is negligible at temperatures above $700 \mathrm{~K}$. It was found that electrical resistivity was heightened somewhat with more Ni interstitial point defects.

Muta et al. (Ref 60) demonstrated that TiNiSn naturally decomposes at $1073 \mathrm{~K}$ during annealing, producing impurity phases such as $\mathrm{TiNi}_{2} \mathrm{Sn}$, Ti-Sn binary phases, and $\mathrm{Sn}$. This highlights the usefulness of a high pressure, densification step toward producing purer TiNiSn. They also showed that sintering from 1273 to $1473 \mathrm{~K}$ with applied pressure using SPS eliminates the majority of the impurity phases. Increasing the SPS temperature further reduces impurities, shown by the sharpening of their XRD peaks. Finally, Douglas et al. (Ref 31) suggest that additional $\mathrm{TiNi}_{2} \mathrm{Sn}$ phase could have a decreasing effect on resistivity at elevated temperatures from excitation from the Heusler and other metallic phases.

Based on the preceding theories and the experimental data from the literature, it can be theorized that regardless of densification or sintering parameters used for stoichiometric TiNiSn, a small amount of $\mathrm{Ni}$ defects sites will always be present, which will increase the electrical resistivity compared to pure TiNiSn. Simultaneously, the ever-present impurity phases seem to have a dominant global effect, since all reported samples have a reduced resistivity compared to the theoretical calculation for TiNiSn.

\subsection{Seebeck Coefficient and Power Factor}

The Seebeck effect produces a voltage in response to a temperature difference across a material, measured as its Seebeck coefficient, $S$. With an applied temperature gradient, high-energy charge carriers (holes or electrons) from the hot portion will diffuse toward the cold portion. Simultaneously, low-energy charge carriers will diffuse to the hot side, but at a slower rate. This produces a charge density gradient, ultimately
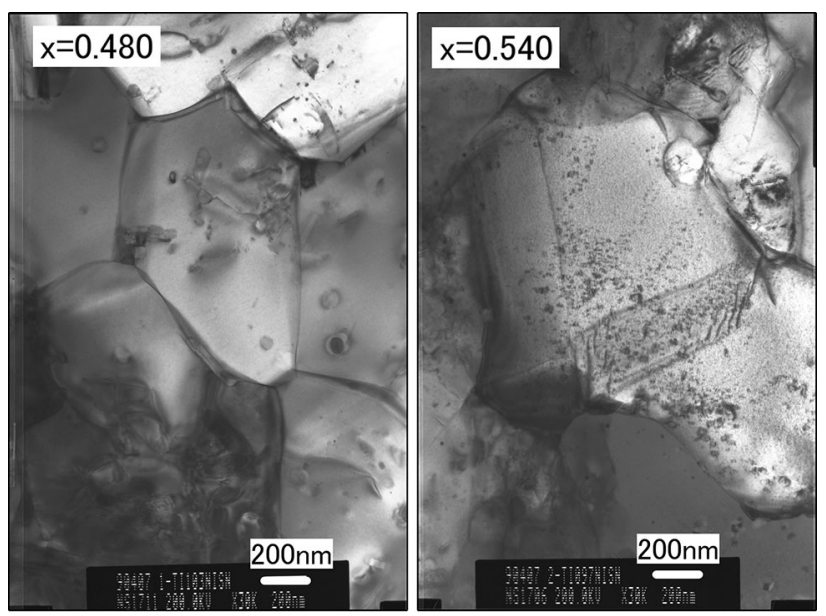

Fig. 7 TEM micrograph of TiNiSn alloy, where $x=\mathrm{Ni} /(\mathrm{Ti}+\mathrm{Sn})$ (Ref 58). reproduced with permission generating an electric field and therefore a voltage. For degenerate metals or semiconductors, the Seebeck coefficient can be given as:

$S=\frac{8 \pi^{2} k_{\mathrm{B}}^{2}}{3 e h^{2}} m^{*} T\left(\frac{\pi}{3 n}\right)^{2 / 3}$

where $m^{*}$ is the effective mass, $n$ is the charge carrier concentration, $k_{\mathrm{B}}$ is the Boltzmann's constant, $e$ is the electron charge, and $h$ is the Planck's constant (Ref 61). Based on Eq 3, as charge carrier concentration increases, $|S|$ will decrease. Since the contribution from holes and electrons interferes with one another in semiconductors, the electrical conductivity of each charge carrier type at a given temperature is also highly relevant to the overall Seebeck coefficient, which can be summarized as a weighted average shown by Eq 4:

$S=\frac{\sigma_{p} S_{p}-\sigma_{n}\left|S_{n}\right|}{\sigma_{p}+\sigma_{n}}$

where $\sigma$ is the electrical conductivity, $p$ refers to holes (positive charge carriers), and $n$ refers to electrons (negative charge carriers) (Ref 62). In general, the charge carrier concentration of TiNiSn is increased through addition of intermetallic impurity phases (i.e., $\mathrm{TiNi}_{2} \mathrm{Sn}$ ) and $\mathrm{Ni}$ defects. The Seebeck coefficient with respect to synthesis parameters and temperature is shown in Fig. 8.

TiNiSn is naturally an intrinsic n-type thermoelectric material, meaning that electrons are the dominant charge carrier and the resulting Seebeck coefficient is negative (Eq 4). Berche and Jund (Ref 54) calculated the theoretical Seebeck coefficient (dashed line), based on a bandgap of $0.12 \mathrm{eV}$. Regardless of the synthesis parameters, the prevalence of $\mathrm{Ni}$ interstitial defects provides additional electron charge carriers, acting as a pseudo-dopant. To maximize $Z T,|S|$ should be maximized (Eq 1). In general for TiNiSn, as temperature increases, $|S|$ increases to a maximum and then decreases. At low temperatures, the ratio of majority to minority charge carriers (i.e., electrons to holes) will be significant. As temperature elevates, the minority charge carrier conductivity increases exponentially, resulting in a peak and decline in $|S|$ (Ref 62). From Fig. 8, it is observed that SPS processes have the lowest $|S|$, followed by HIP and non-densified processes.

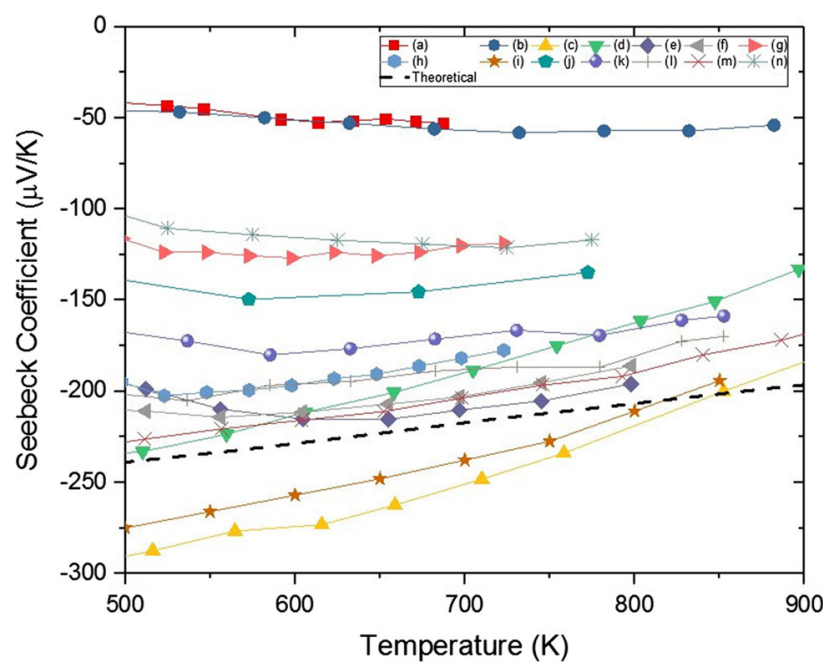

Fig. 8 Seebeck coefficient $(\mu \mathrm{V} / \mathrm{K})$ vs. temperature $(\mathrm{K})$ for selected synthesis parameters of TiNiSn, extracted from the literature 
The higher processing temperature during SPS can allow for high melting point intermetallics to solidify before TiNiSn, producing more impurities and a larger charge carrier concentration.

From the experimental data in Tables 3 and 4, there is a linearly proportional relationship $(p<0.001)$ between the Seebeck coefficient and total electrical resistivity, as illustrated in Fig. 9. This agrees with the theoretical relationship given in Eq 4 and the fact that Seebeck coefficient is inversely proportional to carrier concentration for degenerate materials, such as TiNiSn.

A Jonker plot for TiNiSn, based on the relationship between Seebeck coefficient and logarithm of electrical conductivity, is shown in Fig. 10. For a non-degenerate semiconductor, the Jonker plot relationship is a line that is summarized as:

$S= \pm \frac{k_{\mathrm{B}}}{e}\left(\ln \sigma-\ln \sigma_{0}\right)$

where "+" is for n-type semiconductors and the ideal slope is $86.15 \mu \mathrm{V} / \mathrm{K}(\operatorname{Ref} 63,64)$. The intercept along the $x$-axis, $\ln \left(\sigma_{0}\right)$, is largely determined by the product of the density of states and charge carrier mobility (Ref 65). Deviation from $k_{\mathrm{B}} / e$ in Fig. 10 as temperature increases highlights the natural degeneracy of TiNiSn from Ni-based defects, as electrons are the major charge carrier while $E_{g}$ theoretically shrinks with increasing temperature (Ref 66, 67). The slope reaches a maximum and following decline, corresponding to activation of minority charge carriers. The maximum of the slope matches well with the maximum of $|S|$. Natural logarithm of $\sigma_{0}$ shows small evidence of an increase with temperature, although with very large standard deviation, indicating that carrier mobility of TiNiSn may increase with temperature (Ref 68). Tang et al. (Ref 59) experimentally found that electron mobility reached a maximum at approximately $500 \mathrm{~K}$ for $\mathrm{TiNiSn}_{0.99}$, before the onset of intrinsic excitation of the minority charge carriers as temperature increased. Additionally, Tang et al. found that mobility decreased with increased $\mathrm{Ni}$ content, which is mentioned to change with processing conditions (Ref 59). Therefore, the variance of slope and $\ln \left(\sigma_{0}\right)$ at given temperatures in Fig. 10 can be attributed to the metallic impurity content (Table 2) from each synthesis

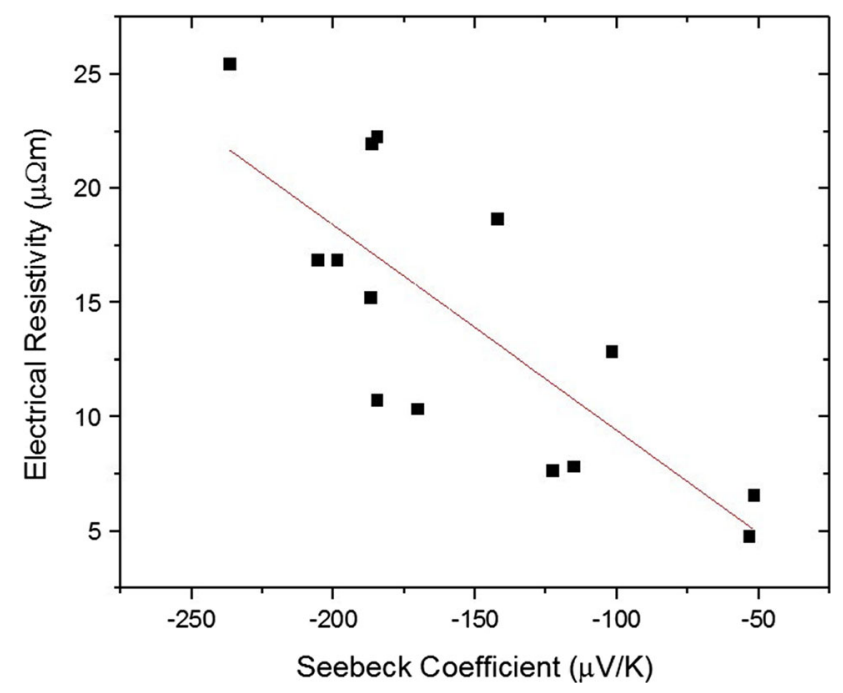

Fig. 9 Average electrical resistivity $(\mu \Omega \mathrm{m})$ vs. Seebeck coefficient $(\mu \mathrm{V} / \mathrm{K})$ of selected synthesis parameters of TiNiSn from 600 to $800 \mathrm{~K}$ method, leading to changes in bandgap, carrier concentration, and charge mobility (Ref 69).

Seebeck coefficient and electrical resistivity are commonly combined into a single term called the power factor, $S^{2} / \rho$, as shown in Fig. 11. Theoretical power factor is calculated based on experimental data from Fig. 5 and 8.

Calculated power factor trends of TiNiSn are typical of degenerate thermoelectric semiconductors since they linearly increase with temperature to a maximum peak, with a subsequent decrease (Ref 62). At elevated temperatures, materials with small resistivity tend to have a low $|S|$, and vice versa. Both the Seebeck coefficient and the resistivity relate to the bandgap, or activation energy needed for electrons to excite into the conduction band. TiNiSn samples with a small resistivity theoretically have a smaller bandgap, which allows easy promotion of electrons to the conduction band at high temperatures. Consequentially, this can excite the holes in the valence band that are available for charge transport, a competing contribution to the Seebeck coefficient. Materials with a larger resistivity, while still affected by the excitation of minority charge carriers, have a larger $|S|$ since the activation energy required for excitation is more substantial (Ref 24). Overall, balancing electrical resistivity and thereby the Seebeck coefficient through processing parameters is essential to maximize power factor and $Z T$.

\subsection{Processing and Thermoelectric Property Relationships}

For metals and degenerate semiconductors, electronic thermal conductivity $\left(k_{E}\right)$ and electrical conductivity have a direct correlation to one another based on the WiedemannFranz law:

$k_{\mathrm{E}}=\sigma L T$

where $L$ is the Lorenz number $\left[L_{0}=2.45 \times 10^{-8} \mathrm{~W} \Omega \mathrm{K}^{-2}\right.$ (Ref 70)] and $\sigma$ is the electrical conductivity. This law holds true and $L_{0}$ is a universal factor when the free electron contribution for heat transport is significantly higher than by phonon transport. For semiconductors and other materials with contributions from both free electrons and phonons, Kim et al. (Ref 71) suggested a new approximation (accurate within 20\%) of $L$ in order to consider phonon scattering:

$L=1.5+\exp \left[-\frac{|S|}{116}\right]$

where $L\left(10^{-8} \mathrm{~W} \Omega \mathrm{K}^{-2}\right)$ is a first-order factor of Seebeck coefficient $(\mu \mathrm{V} / \mathrm{K})$. Since $k_{\text {tot }}=k_{\mathrm{E}}+k_{\mathrm{L}}$, where $k_{\mathrm{L}}$ is the lattice thermal conductivity, $k_{\text {tot }}$ is the experimentally measured total thermal conductivity (Fig. 2), and $k_{\mathrm{E}}$ is calculated from estimated $L$ and measured $S$ (Eq 6 and 7), lattice and electronic contributions to thermal conductivity can be separately estimated for TiNiSn, as illustrated in Fig. 12.

From Fig. 12, it is confirmed that the thermal conductivity is dominated by lattice contributions, comparing well with firstprinciples calculations by Ding et al. (Ref 49). Lattice thermal conductivity is generally increased with a decrease in phonon scattering occurrences, accomplished through increased density (i.e., decreased porosity), fewer grain boundaries, and fewer lattice defects (e.g., Ni defects and impurity phases). Ni lattice defects increase the average atomic mass of localized areas in the bulk TiNiSn crystal, decreasing the lattice thermal conductivity (Ref 72). Complex crystal structures are also associated 


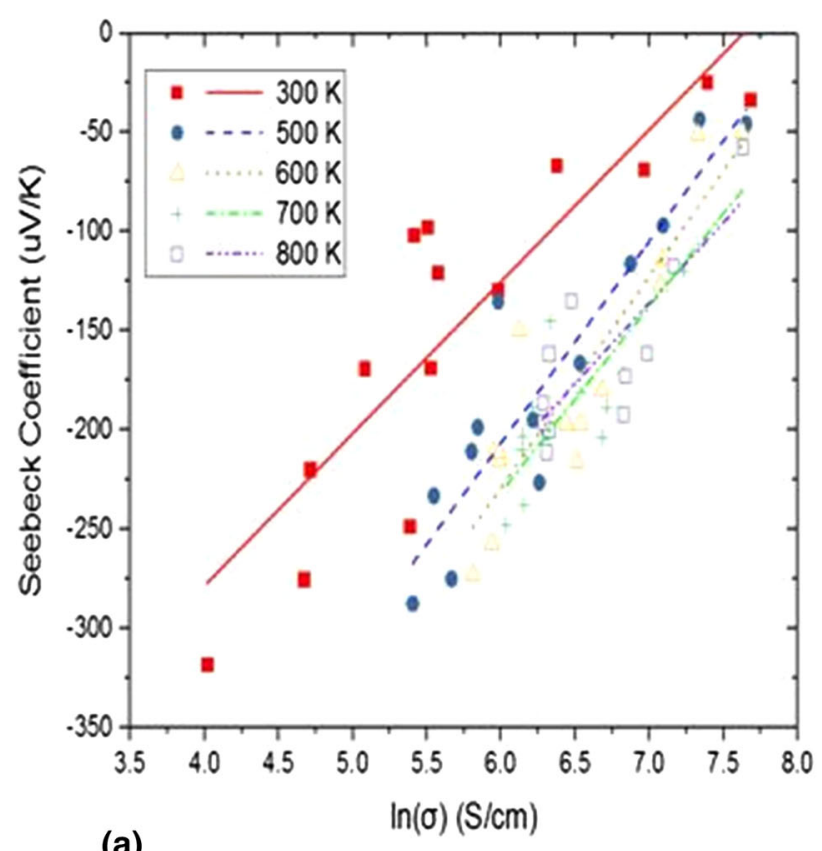

(a)

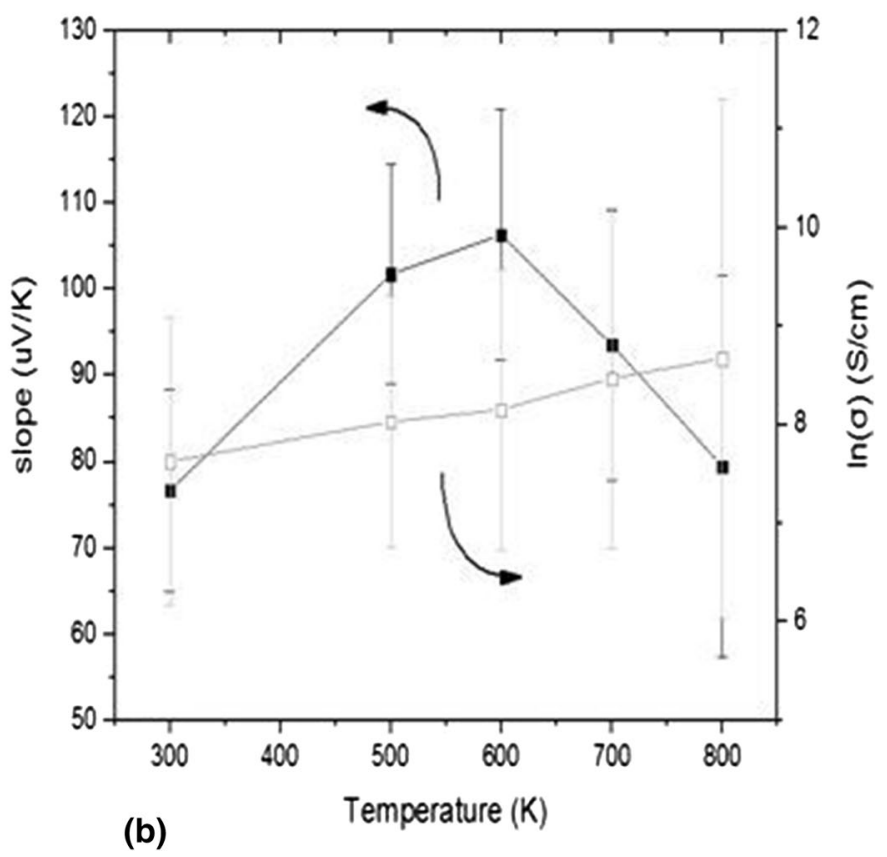

(b)

Fig. 10 (a) Jonker plot of TiNiSn and (b) the slopes and $x$-axis intersections of Jonker plot at different temperatures

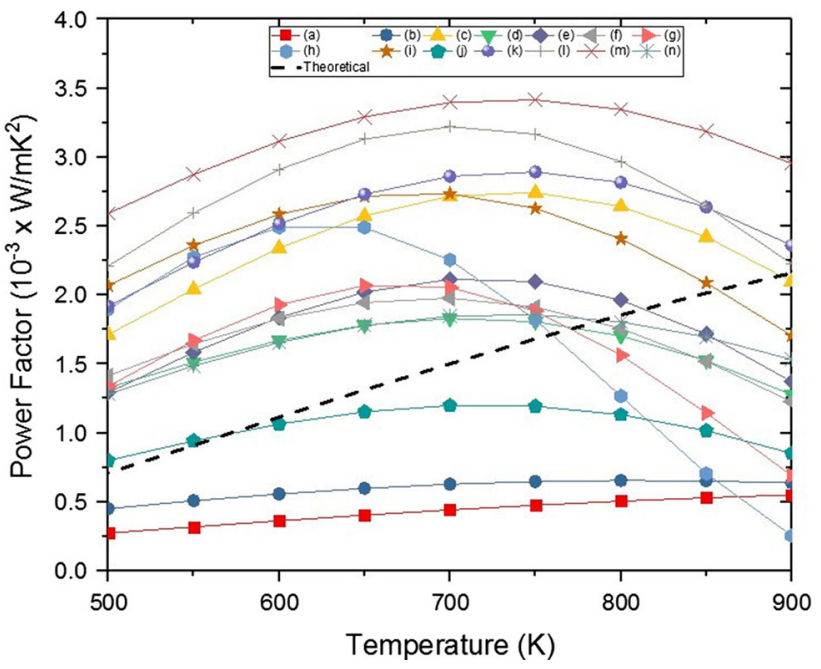

Fig. 11 Power factor $\left(10^{-3} \times \mathrm{W} / \mathrm{m} \mathrm{K}^{2}\right)$ vs. temperature $(\mathrm{K})$ for selected synthesis parameters of TiNiSn, fitted from extracted literature data

with a low lattice thermal conductivity (Ref 73). Alternatively, thermal conductivity through free electron contribution is primarily increased with a greater charge carrier concentration and metallic impurity phase content, such as $\mathrm{TiNi}_{2} \mathrm{Sn}$ (Ref 74).

Based on preceding theories and reported literature data, there are two simultaneous, but opposing factors resulting from processing conditions that influence the overall thermal conductivity: phase and lattice deviations from a pure TiNiSn matrix and density variations (i.e., porosity content). Lower temperature and longer duration annealing methods without densification, such as $c$ and $i$, provide a smaller stable chemical range for TiNiSn (Ref 59), reducing the amount of interstitial $\mathrm{Ni}$ as well as allowing for more time to reach phase equilibrium with fewer metallic phases. The other processes that include a densification step, such as HIP or SPS, are generally done at higher temperatures than annealing, which allow for higher

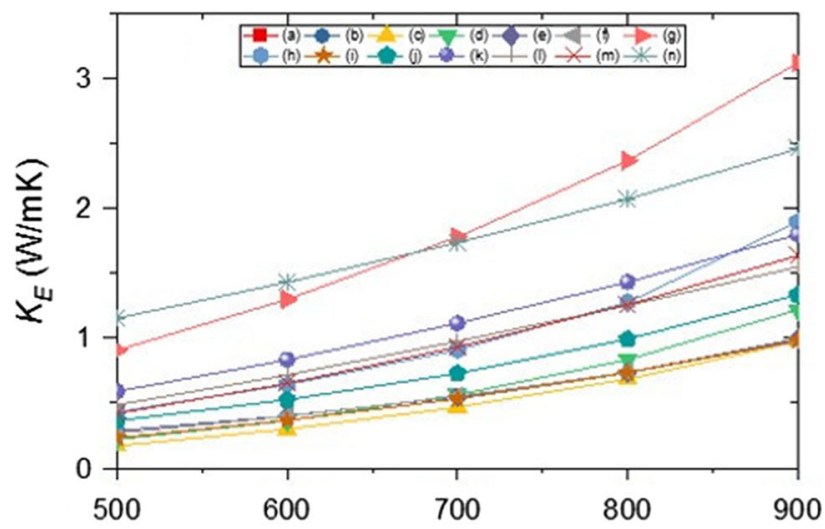

(a)

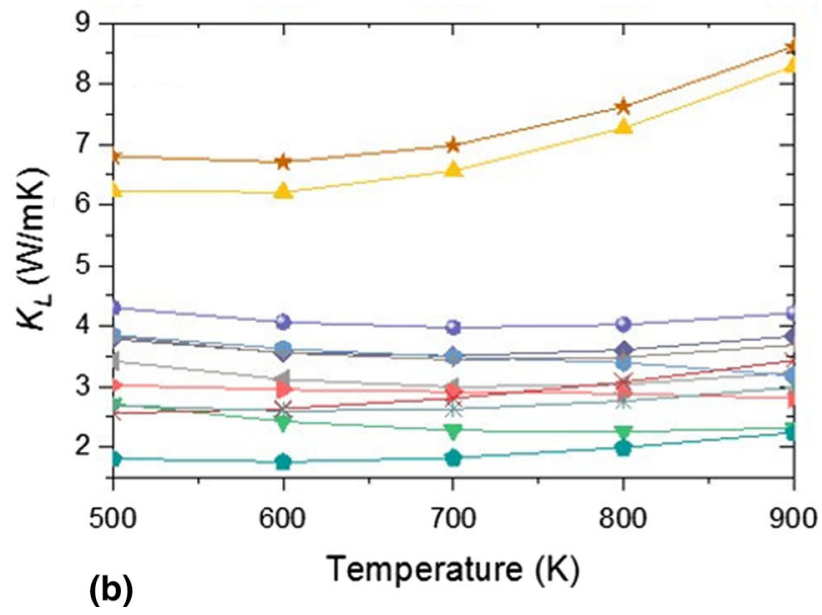

Fig. 12 Decoupled (a) electronic thermal conductivity, $k_{\mathrm{e}}$, and (b) lattice thermal conductivity, $k_{\mathrm{L}}$, vs. temperature $(\mathrm{K})$ for selected synthesis parameters of TiNiSn 
melting point impurity phases (e.g., $\mathrm{TiNi}_{2} \mathrm{Sn}$ ) to solidify preferentially. Additionally, higher temperatures allow for increased amounts of interstitial $\mathrm{Ni}$ defects due to the widening stable chemical window. This is reflected in Fig. 2, 8, and 12, where high-temperature densification processes have much lower lattice thermal conductivity (as much as 58\% (Ref 59)), heightened electronic thermal conductivity, and low Seebeck coefficient, presumably due to increased charge carrier concentration from such defects.

Density and porosity were previously correlated with total thermal conductivity in an earlier section. It was also found that SPS was the optimal densification process for fully dense bulk samples, when compared to HIP. Since larger densities promote less scattering to occur, SPS generally produced samples with larger total thermal conductivity. Defect content is the apparent major factor influencing both conductivity contributions, since processes $c$ and $i$ have the largest lattice conductivity and smallest electronic component from Fig. 12. These two processes were also the sole sources to report a quantitatively high TiNiSn matrix purity ( $97-98 \%)$. While one can speculate that SPS processes (i.e., $g$ and $n$ ) have the largest content of $\mathrm{Ni}$ defects and/or impurity phases since they have the largest electronic thermal conductivity, it is obvious that the lack of quantitative phase analysis in the literature leaves a gap toward a complete understanding.

Since electrical resistivity is directly related to thermal conductivity through the Lorenz number, $L$, it is also affected by sample density and defect microstructure. Ni defects can be considered interstitial point defects, which traditionally increase electrical resistivity as:

$\Delta \rho=N c^{\mathrm{a}} \rho_{\mathrm{p}}$

where $N$ is the number of lattice sites, $c^{\mathrm{a}}$ is the concentration of point defects, and $\rho_{\mathrm{p}}$ is the resistivity increase per point defect (Ref 37). Additionally, the effective resistivity of dispersed secondary phases in a surrounding matrix can be estimated using a mixture rule (Ref 75$)$. With two outliers omitted, there is an obvious inverse correlation ( $p=0.036)$, as illustrated in Fig. 13. The two outliers correspond to processes $c$ and $i$, which do not include a densification step and produce a unique combination of low density, high grain size, assumed higher phase purity, and low Ni defect concentration. When looking at more comparable processes utilizing either HIP or SPS, lower density HIP samples had the lower thermal conductivities and predictably larger electrical resistivities. This is due to more electron scattering effects from higher porosity contents. Electrical resistivity is negatively influenced by the metallic impurity content through a smaller bandgap in addition to increased electronic contributions to $k_{L}(\operatorname{Ref} 74)$, but again, more quantitative phase data are required to compare.

While Seebeck coefficient is traditionally associated with purely electronic behavior and is significantly correlated with electrical resistivity (Fig. 9, 10), sample porosity may have a minor effect on Seebeck coefficient. Lee et al. (Ref 76) found that nano-sized pores in SiGe not only reduced thermal conductivity and electrical conductivity, but also increased the Seebeck coefficient. Valalaki et al. (Ref 77) found a similar effect in porous silicon (up to $\sim 50 \%$ porosity). This was attributed to an energy filtering effect where small pores will scatter low-energy electrons more effectively than high-energy electrons. Since $S$ is essentially a measure of average energy of

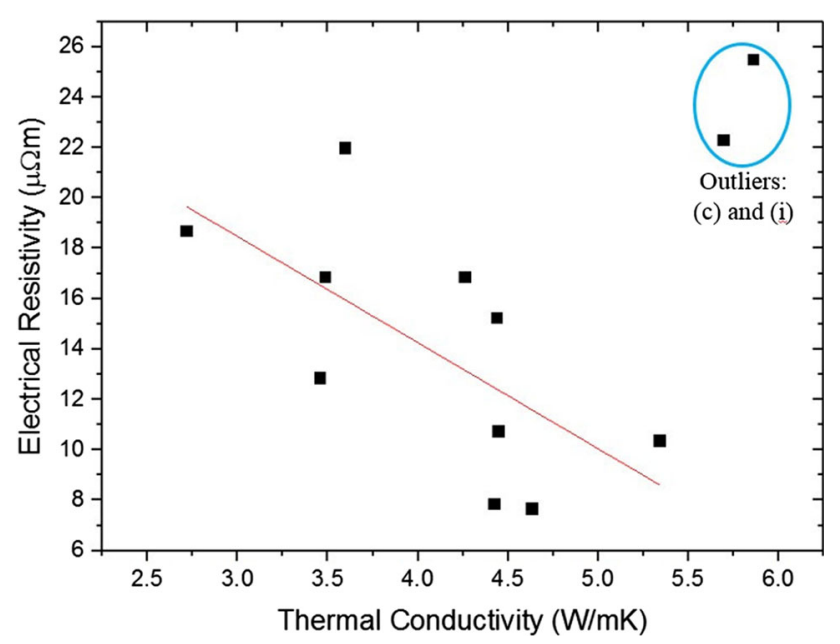

Fig. 13 Average electrical resistivity $(\mu \Omega \mathrm{m})$ vs. total thermal conductivity $(\mathrm{W} / \mathrm{m} \mathrm{K})$ of selected synthesis parameters of TiNiSn from 600 to $800 \mathrm{~K}$

electrons, as the pore content and proportion of high-energy electrons go up, so does $S$. Nano- and micro-sized pores are observed in TiNiSn (Ref 31, 34, 35, 38, 48) through various microscopy techniques. While charge carrier density and secondary phase content are surely the most significant factor concerning $S$, pore content could affect $S$ of TiNiSn to some degree, although statistical significance of porosity cannot be confirmed with the available literature.

\subsection{Figure of Merit}

From Eq 1, ZT is a function of the Seebeck coefficient, electrical resistivity, thermal conductivity, and temperature. Given the collected data from Fig. 2, 5, and 8, the theoretical and experimental $Z T$ as a function of temperature and synthesis process can be calculated as shown in Fig. 14.

Regardless of synthesis parameters, the $Z T$ of TiNiSn increases with rising temperature to a peak $\left(T_{\text {avg }} \approx 750 \mathrm{~K}\right)$, after which $Z T$ decreases. The majority of synthesis processes produce a maximum $Z T$ between 0.3 and 0.4 , with some outliers outside of this range. Process $a$ (and presumably $b$ ) produced very low $Z T$ due to the resulting metallic nature, based on calculated bandgap. Processes $m$ and $l$ have significantly higher $Z T$ than most other techniques, attributed to their below average thermal conductivity and high power factor. There is not an apparent relationship of synthesis parameter versus $Z T$, nor of $Z T_{\max }$ versus $T_{\max }(p>0.15)$, as shown in Fig. 14, concluding that it is important to balance each interconnected thermoelectric property when optimizing $Z T$.

\subsection{Theoretical and Experimental Differences}

There are significant differences between the calculated properties and the available experimental data, which can partly be explained by the assumptions made. Accurate determination of grain size and grain boundary morphology has a great influence on thermal conductivity, since the lattice contribution is most significant up to $800 \mathrm{~K}$ ( $\operatorname{Ref} 49,54)$. Also, bandgap of synthesized TiNiSn is smaller than theoretical calculations, which effects electron promotion behavior, electrical resistivity, and the Seebeck coefficient (Ref 52, 53). Additionally, Berche 


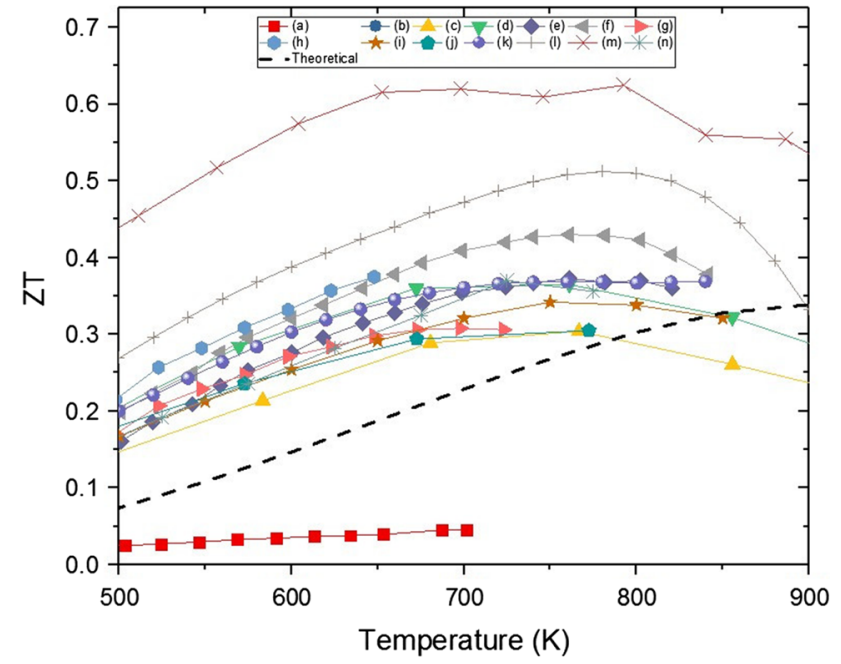

Fig. $14 Z T$ vs. temperature $(\mathrm{K})$ for selected synthesis parameters of TiNiSn, extracted from the literature

and Jund (Ref 54) assumed a constant electron relaxation time, $\tau$, which is inversely proportional to electrical resistivity (Ref 78 ). For a semiconductor, $\tau$ should theoretically increase with temperature.

Variations in phase morphology between synthesized and theoretically pure TiNiSn should also be a primary source of differences within each property. Based on the previously mentioned difficulty of synthesizing pure TiNiSn, small amounts of impurity phases $\left(\mathrm{TiNi}_{2} \mathrm{Sn}, \mathrm{Sn}\right.$, etc.) and inherent, interstitial $\mathrm{Ni}$ defects will change the majority and minority charge carrier concentration ratio, charge carrier concentration, and therefore the thermoelectric properties (Ref 31, 79, 80). Lack of reported quantitative phase analysis is the primary source of uncertainty when refining process/property relationships. Berche and Jund (Ref 81) also predict that small amounts of oxygen can decompose the TiNiSn phase into $\mathrm{Ti}_{2} \mathrm{O}_{3}$ and $\mathrm{Ni}_{3} \mathrm{Sn}_{2}+\mathrm{Ni}_{3} \mathrm{Sn}_{4}$, decreasing thermal conductivity, Seebeck coefficient, and electrical resistivity. Ultimately, the reported theoretical calculations provide a somewhat accurate global trend for TiNiSn, but differences due to synthesis difficulties and calculation assumptions should be taken into account for continued evaluation and prediction of half-Heusler thermoelectric properties.

\section{Conclusions}

TiNiSn thermoelectric properties have been reported based on a variety of melting and sintering technique combinations, comprehensively compared for the first time in this review. Since many plausible applications for TiNiSn are at elevated temperature, the thermoelectric properties from approximately 600 to $800 \mathrm{~K}$ were focused on. Theoretical calculations of the thermoelectric properties exhibit significant variation from reported data due to assumptions regarding phase purity, morphology, theoretical density, and electronic structure. Primary melting techniques, such as arc melting and induction levitation melting, do not seem to have a significant effect on thermoelectric properties.
Total thermal conductivity $(2.47-6.08 \mathrm{~W} / \mathrm{m} \mathrm{K})$ was found to be dominated by phonon scattering effects, when separated into lattice and electronic contributions. While a direct correlation was found between total thermal conductivity and process density (i.e., porosity), charge carrier concentration will also contribute. Methods utilizing SPS produced densities closer to $100 \%$ and higher total thermal conductivities compared to HIP methods due to less phonon scattering effects. Additionally, densification methods are done at comparatively higher temperatures, which widens the phase window of TiNiSn for $\mathrm{Ni}$ defects to occur and also allows for intermetallic phases to preferentially solidify and stabilize due to higher melting temperatures. Although, some non-densification processes had lower densities, large grain size and fewer lattice scattering events from impurities and $\mathrm{Ni}$ defects $(97-98 \%$ pure TiNiSn) allowed for large thermal conductivities.

Electrical resistivity $(4.75-30 \mu \Omega \mathrm{m})$ is inversely related to thermal conductivity, since scattering detrimentally affects both free electron mobility and phonon transport. Utilizing SPS produces higher densities and therefore lower electrical resistivity, compared to HIP. Since all reported alloys have intermetallic impurities, there is a global trend of lower electrical resistivity compared to theoretical calculations due to increased charge carrier concentration. It can be predicted based on measured electrical resistivity and processing temperature that phase defect quantity increases when utilizing densification techniques.

Seebeck coefficient $(-273.20$ to $-50.71 \mu \mathrm{V} / \mathrm{K})$ is directly correlated with electrical resistivity, primarily due to a similar relationship with charge carrier concentration. Quantification of this relationship is not possible since phase percentages are not provided in the literature. Density and porosity could be minor effects on the Seebeck coefficient, as nano- and micro-pores were observed in TiNiSn, although significant correlation was not proven based on the current literature. Additions of nanosized pores have been previously observed to scatter lowenergy electrons, which cause increases in Seebeck coefficient and electrical resistivity. The largest Seebeck coefficients were found when densification techniques were not used, followed by smaller values with HIP and SPS, respectively.

Interestingly, the processes that produced the largest maximum ZT (0.046-0.625) are those that have an intermediate values for all thermoelectric properties, since many inverse relationships exist. The ideal thermoelectric TiNiSn alloy has near theoretical density, small amounts of metallic impurities and interstitial $\mathrm{Ni}$, and a small grain size, achievable through a multistep process involving (1) initial melting of elemental components, (2) annealing heat treatment to reduce phase impurities, and (3) densification through SPS or HIP to reduce grain size.

While thermoelectric properties are justifiably focused on in the majority of publications, SEM/EDS micrographs and quantitative phase analysis are rarely provided, leaving a gap in understanding. Additional phase information would be a critical statistic toward separating contributions of Ni defects and intermetallic impurity phases toward TE properties. Future research endeavors on TiNiSn alloys and half-Heuslers should strive to provide more detailed, standardized chemical and microstructural information regarding impurity phase formation in order to further compare the unique qualities of each synthesis procedure and their impact on the optimization of thermoelectric properties. 


\section{Acknowledgment}

The authors acknowledge the financial support provided by NSF, ACIPCO, and the Metallurgical and Materials Engineering Department at The University of Alabama.

\section{References}

1. H.J. Goldsmid, The Thermoelectric and Related Effects, Introduction to Thermoelectricity, 2nd ed., R. Hull et al., Eds., Berlin, Heidelberg, Springer, 2016, p 1-7. https://doi.org/10.1007/978-3-662-49256-7_1

2. L.E. Bell, Cooling, Heating, Generating Power, and Recovering Waste Heat with Thermoelectric Systems, Science (80), 2008, 321(5895), p 1457-1461. https://doi.org/10.1126/science.1158899

3. M.R. Bogala, Synthesis, Characterization, and Phase Stability of High Temperature Thermoelectric Metal Borides and Silicides, The University of Alabama, Tuscaloosa, 2016

4. A.M. Dehkordi, M. Zebarjadi, J. He, and T.M. Tritt, Thermoelectric Power Factor: Enhancement Mechanisms and Strategies for Higher Performance Thermoelectric Materials, Mater. Sci. Eng. R Rep., 2015, 97, $\mathrm{p} 1-22$

5. S. Hébert, D. Berthebaud, R. Daou, Y. Bréard, D. Pelloquin, E. Guilmeau, F. Gascoin, O. Lebedev, and A. Maignan, Searching for New Thermoelectric Materials: Some Examples among Oxides, Sulfides and Selenides, J. Phys.: Condens. Matter, 2015, 28(1), p 13001

6. S. Chen and Z. Ren, Recent Progress of Half-Heusler for Moderate Temperature Thermoelectric Applications, Mater. Today, 2013, 16(10), p 387-395

7. H. Goldsmid, Bismuth Telluride and Its Alloys as Materials for Thermoelectric Generation, Materials (Basel)., 2014, 7(4), p 2577 2592

8. M. Rull-Bravo, A. Moure, J.F. Fernandez, and M. Martín-González, Skutterudites as Thermoelectric Materials: Revisited, RSC Adv., 2015, 5(52), p 41653-41667

9. M.R. Bogala and R.G. Reddy, Reaction Kinetic Studies of MetalDoped Magnesium Silicides, J. Mater. Sci., 2017, 52(20), p 11962 11976

10. S.K. Bux, M.T. Yeung, E.S. Toberer, G.J. Snyder, R.B. Kaner, and J.-P. Fleurial, Mechanochemical Synthesis and Thermoelectric Properties of High Quality Magnesium Silicide, J. Mater. Chem., 2011, 21(33), p 12259-12266

11. L. Huang, Q. Zhang, B. Yuan, X. Lai, X. Yan, and Z. Ren, Recent Progress in Half-Heusler Thermoelectric Materials, Mater. Res. Bull., 2016, 76, p 107-112

12. C. Fu, S. Bai, Y. Liu, Y. Tang, L. Chen, X. Zhao, and T. Zhu, Realizing High Figure of Merit in Heavy-Band p-Type Half-Heusler Thermoelectric Materials, Nat. Commun., 2015, 6, p 8144

13. S. Walia, S. Balendhran, H. Nili, S. Zhuiykov, G. Rosengarten, Q.H. Wang, M. Bhaskaran, S. Sriram, M.S. Strano, and K. Kalantar-zadeh, Transition Metal Oxides-Thermoelectric Properties, Prog. Mater Sci., 2013, 58(8), p 1443-1489

14. B. Yu, M. Zebarjadi, H. Wang, K. Lukas, H. Wang, D. Wang, C. Opeil, M. Dresselhaus, G. Chen, and Z. Ren, Enhancement of Thermoelectric Properties by Modulation-Doping in Silicon Germanium Alloy Nanocomposites, Nano Lett., 2012, 12(4), p 2077-2082

15. L. Bjerg, G.K.H. Madsen, and B.B. Iversen, Enhanced Thermoelectric Properties in Zinc Antimonides, Chem. Mater, 2011, 23(17), p 3907 3914

16. J.-W.G. Bos and R.A. Downie, Half-Heusler Thermoelectrics: A Complex Class of Materials, J. Phys.: Condens. Matter, 2014, 26(43), p 433201

17. R. Stern, B. Dongre, and G.K.H. Madsen, Extrinsic Doping of the Half-Heusler Compounds, Nanotechnology, 2016, 27(33), p 334002

18. S. Poon, Recent Advances in Thermoelectric Performance of HalfHeusler Compounds, Metals (Basel), 2018, 8(12), p 989

19. M. Gürth, A. Grytsiv, J. Vrestal, V.V. Romaka, G. Giester, E. Bauer, and P. Rogl, On the Constitution and Thermodynamic Modelling of the System Ti-Ni-Sn, RSC Adv., 2015, 5(112), p 92270-92291

20. M. Imam, J.S Young, and R.G. Reddy, Determination of Thermodynamic Properties of Si-B Alloys, Metall. Mater. Trans. B 2019, 50(2), p 981-990
21. M.A. Imam and R.G. Reddy, Thermodynamic Properties of Magnesium-Boron Binary Alloys Determined Using Solid-State Electrochemical Measurements, Metall. Mater. Trans. B, 2018, 49(6), p 3504 3512

22. M.R. Bogala and R.G. Reddy, Metal Silicides for High-Temperature Thermoelectric Application, Applications of Process Engineering Principles in Materials Processing, Energy and Environmental Technologies, S. Wang et al., Eds., Berlin, Springer, 2017, p 421-434

23. R.A. Downie, D.A. MacLaren, R.I. Smith, and J.W.G. Bos, Enhanced Thermoelectric Performance in TiNiSn-Based Half-Heuslers, Chem. Commun., 2013, 49(39), p 4184-4186

24. J.S. Young, Synthesis and Characterization of $\mathrm{TiNi}_{(1+x)} \mathrm{Sn}$ Thermoelectric Alloys, University of Alabama Libraries, Tuscaloosa, 2017

25. W. Gierlotka and S. Chen, Thermodynamic Descriptions of the $\mathrm{Cu}-\mathrm{Zn}$ System, J. Mater. Res., 2008, 23(1), p 258-263

26. E.C. Okress, D.M. Wroughton, G. Comenetz, P.H. Brace, and J.C.R. Kelly, Electromagnetic Levitation of Solid and Molten Metals, J. Appl. Phys., 1952, 23(5), p 545-552

27. R. Reddy, S. Pedneker, and P. Rao, Kinetics of Decarburization of FeC-Cr Alloys, Trans. Ind. Inst. Metals, 1976, 29(6), p 426-431

28. S.I. Bakhtiyarov and D.A. Siginer, Electromagnetic Levitation, Part II: Thermophysical Property Measurements in Terrestrial Conditions, FDMP Fluid Dyn. Mater. Process., 2008, 4(3), p 163-184

29. Z. Royer, Optimizing Electromagnetic Levitation Design to Enhance Thermodynamic Measurement, Digital Repository @ Iowa State University, Iowa, 2012

30. N. Verma, J.E. Douglas, S. Krämer, T.M. Pollock, R. Seshadri, and C.G. Levi, Microstructure Evolution of Biphasic $\mathrm{TiNi}_{1+x} \mathrm{Sn}$ Thermoelectric Materials, Metall. Mater. Trans. A, 2016, 47(8), p 4116-4127

31. J.E. Douglas, C.S. Birkel, M.-S. Miao, C.J. Torbet, G.D. Stucky, T.M. Pollock, and R. Seshadri, Enhanced Thermoelectric Properties of Bulk TiNiSn via Formation of a $\mathrm{TiNi}_{2} \mathrm{Sn}$ Second Phase, Appl. Phys. Lett., 2012, 101(18), p 183902

32. H.J. Kitchen, S.R. Vallance, J.L. Kennedy, N. Tapia-Ruiz, L. Carassiti, A. Harrison, A.G. Whittaker, T.D. Drysdale, S.W. Kingman, and D.H. Gregory, Modern Microwave Methods in Solid-State Inorganic Materials Chemistry: From Fundamentals to Manufacturing, Chem. Rev., 2013, 114(2), p 1170-1206

33. K. Biswas, S. Muir, and M.A. Subramanian, Rapid Microwave Synthesis of Indium Filled Skutterudites: An Energy Efficient Route to High Performance Thermoelectric Materials, Mater. Res. Bull., 2011, 46(12), p 2288-2290

34. Y. Lei, Y. Li, L. Xu, J. Yang, R. Wan, and H. Long, Microwave Synthesis and Sintering of TiNiSn Thermoelectric Bulk, J. Alloys Compd., 2016, 660, p 166-170

35. C.S. Birkel, W.G. Zeier, J.E. Douglas, B.R. Lettiere, C.E. Mills, G. Seward, A. Birkel, M.L. Snedaker, Y. Zhang, G.J. Snyder et al., Rapid Microwave Preparation of Thermoelectric TiNiSn and TiCoSb HalfHeusler Compounds, Chem. Mater., 2012, 24(13), p 2558-2565

36. P. George, S.C. Parida, and R.G. Reddy, Thermodynamic Studies on the System Nb-Al, Metall. Mater. Trans. B, 2007, 38(1), p 85-91

37. G. Gottstein, Physical Foundations of Materials Science, Springer, Berlin, 2004

38. Y. Gelbstein, N. Tal, A. Yarmek, Y. Rosenberg, M.P. Dariel, S. Ouardi, B. Balke, C. Felser, and M. Köhne, Thermoelectric Properties of Spark Plasma Sintered Composites Based on TiNiSn Half-Heusler Alloys, $J$. Mater. Res., 2011, 26(15), p 1919-1924

39. Z. Shen, M. Johnsson, Z. Zhao, and M. Nygren, Spark Plasma Sintering of Alumina, J. Am. Ceram. Soc., 2002, 85(8), p 1921-1927

40. M. Suárez, A. Fernández, J.L. Menéndez, R. Torrecillas, H.U. Kessel, J. Hennicke, R. Kirchner, and T. Kessel, Challenges and Opportunities for Spark Plasma Sintering: A Key Technology for a New Generation of Materials, Sintering Applications, B. Ertuğ, Ed., IntechOpen, 2013, p 319-342

41. R.G. Reddy, A.M. Yahya, and L. Brewer, Thermodynamic Properties of Ti-Al Intermetallics, J. Alloys Compd., 2001, 321(2), p 223-227

42. F.B. Swinkels, D.S. Wilkinson, E. Arzt, and M.F. Ashby, Mechanisms of Hot-Isostatic Pressing, Acta Metall., 1983, 31(11), p 1829-1840

43. G. Delaizir, G. Bernard-Granger, J. Monnier, R. Grodzki, O. Kim-Hak, P.-D. Szkutnik, M. Soulier, S. Saunier, D. Goeuriot, O. Rouleau et al., A Comparative Study of Spark Plasma Sintering (SPS), Hot Isostatic Pressing (HIP) and Microwaves Sintering Techniques on p-Type $\mathrm{Bi}_{2} \mathrm{Te}_{3}$ Thermoelectric Properties, Mater. Res. Bull., 2012, 47(8), p 1954-1960 
44. A. Yegyan Kumar, Effects of Hot Isostatic Pressing on Copper Parts Additively Manufactured via Binder Jetting. Virginia Tech, 2018

45. G. Byrne, M.A. Spence, B. Olsen, P.J. Houghton, and J. McMahon, Advantages of Hot Isostatic Pressing (HIP) Production Routes for Process Manifolds, Duplex Stainless Steels'94, in Proceeding of International Conference on Duplex Stainless Steels, Glasgow, Scotland, Cambridge TWI, paper, 1994

46. K. Essa, R. Khan, H. Hassanin, M.M. Attallah, and R. Reed, An Iterative Approach of Hot Isostatic Pressing Tooling Design for NetShape IN718 Superalloy Parts, Int. J. Adv. Manuf. Technol., 2016, 83(9-12), p 1835-1845

47. R.M. German, P. Suri, and S.J. Park, Liquid Phase Sintering, J. Mater. Sci., 2009, 44(1), p 1-39

48. Y. Kimura, C. Asami, Y.W. Chai, and Y. Mishima, Thermoelectric Performance of Half-Heusler TiNiSn Alloys Fabricated by SolidLiquid Reaction Sintering, Mater. Sci. Forum, 2010, 654, 2795-2798

49. G. Ding, G.Y. Gao, and K.L. Yao, Examining the Thermal Conductivity of the Half-Heusler Alloy TiNiSn by First-Principles Calculations, J. Phys. D Appl. Phys., 2015, 48(23), p 235302

50. M. Schrade, K. Berland, S.N.H. Eliassen, M.N. Guzik, C. EchevarriaBonet, M.H. Sørby, P. Jenuš, B.C. Hauback, R. Tofan, A.E. Gunnæs et al., The Role of Grain Boundary Scattering in Reducing the Thermal Conductivity of Polycrystalline XNiSn (X = Hf, Zr, Ti) Half-Heusler Alloys, Sci. Rep., 2017, 7(1), p 13760

51. S. Bhattacharya, T.M. Tritt, Y. Xia, V. Ponnambalam, S.J. Poon, and N. Thadhani, Grain Structure Effects on the Lattice Thermal Conductivity of Ti-Based Half-Heusler Alloys, Appl. Phys. Lett., 2002, 81(1), p 43-45

52. H. Hazama, R. Asahi, M. Matsubara, and T. Takeuchi, Study of Electronic Structure and Defect Formation in $\mathrm{Ti}_{1-x} \mathrm{Ni}_{1+x} \mathrm{Sn}$ HalfHeusler Alloys, J. Electron. Mater., 2010, 39(9), p 1549-1553

53. S. Öğüt and K.M. Rabe, Band Gap and Stability in the Ternary Intermetallic Compounds NiSnM (M = Ti, Zr, Hf): A First-Principles Study, Phys. Rev. B, 1995, 51(16), p 10443

54. A. Berche and P. Jund, Fully Ab-Initio Determination of the Thermoelectric Properties of Half-Heusler NiTiSn: Crucial Role of Interstitial Ni Defects, Materials (Basel), 2018, 11(6), p 868

55. R.A. Downie, S.R. Popuri, H. Ning, M.J. Reece, and J.-W.G. Bos, Effect of Spark Plasma Sintering on the Structure and Properties of $\mathrm{Ti}_{1-\mathrm{x}} \mathrm{Zr}_{\mathrm{x}} \mathrm{NiSn}$ Half-Heusler Alloys, Materials (Basel), 2014, 7(10), p 7093-7104

56. J. Hassel and J. Tervo, Thermoelectric Properties of Sintered N-Type and p-Type Tellurides, J. Electron. Mater, 2013, 42(7), p 1745-1750

57. L. Lu, Y. Shen, X. Chen, L. Qian, and K. Lu, Ultrahigh Strength and High Electrical Conductivity in Copper, Science, 2004, 304(5669), p 422-426

58. H. Hazama, M. Matsubara, R. Asahi, and T. Takeuchi, Improvement of Thermoelectric Properties for Half-Heusler TiNiSn by Interstitial Ni Defects, J. Appl. Phys., 2011, 110(6), p 63710

59. Y. Tang, X. Li, L.H.J. Martin, E.C. Reyes, T. Ivas, C. Leinenbach, S Anand, M. Peters, G.J. Snyder, and C. Battaglia, Impact of Ni Content on the Thermoelectric Properties of Half-Heusler TiNiSn, Energy Environ. Sci., 2018, 11(2), p 311-320

60. H. Muta, T. Yamaguchi, K. Kurosaki, and S. Yamanaka, Thermoelectric Properties of ZrNiSn Based Half Heusler Compounds, in 24th International Conference on Thermoelectrics, 2005. ICT 2005 (2005), p. 351-354

61. S.M. Kauzlarich, S.R. Brown, and G.J. Snyder, Zintl Phases for Thermoelectric Devices, Dalt. Trans., 2007, 21, p 2099-2107

62. Z.M. Gibbs, H.-S. Kim, H. Wang, and G.J. Snyder, Band Gap Estimation from Temperature Dependent Seebeck Measurement—-deviations from the 2e|S|MaxTmax Relation, Appl. Phys. Lett., 2015, 106(2), p 22112

63. G.H. Jonker, The Application of Combined Conductivity and SeebeckEffect Plots for the Analysis of Semiconductor Properties(Conductivity vs Seebeck Coefficient Plots for Analyzing n-Type, p-Type and Mixed Conduction Semiconductors Transport Properties), Philips Res. Reports, 1968, 23, p 131-138

64. H. Muta, T. Kanemitsu, K. Kurosaki, and S. Yamanaka, High-Temperature Thermoelectric Properties of Nb-Doped MNiSn $(\mathrm{M}=\mathrm{Ti}, \mathrm{Zr})$ HalfHeusler Compound, J. Alloys Compd., 2009, 469(1-2), p 50-55

65. Q. Zhu, E.M. Hopper, B.J. Ingram, and T.O. Mason, Combined Jonker and Ioffe Analysis of Oxide Conductors and Semiconductors, J. Am. Ceram. Soc., 2011, 94(1), p 187-193

66. K. Komoto and T. Mori, Thermoelectric Nanomaterials: Materials Design and Applications, Springer, Berlin, 2013
67. Y. Kinemuchi, C. Ito, H. Kaga, T. Aoki, and K. Watari, Thermoelectricity of Al-Doped $\mathrm{ZnO}$ at Different Carrier Concentrations, J. Mater. Res., 2007, 22(7), p 1942-1946

68. J.-I. Jung, S.T. Misture, and D.D. Edwards, Seebeck Coefficient and Electrical Conductivity of $\mathrm{BSCF} \quad\left(\mathrm{Ba}_{0.5} \mathrm{Sr}_{0.5} \mathrm{Co}_{\mathrm{x}} \mathrm{Fe}_{1-\mathrm{x}} \mathrm{O}_{3-\delta}\right.$, $0 \leq \mathrm{x} \leq 0.8)$ as a Function of Temperature and Partial Oxygen Pressure, Solid State Ionics, 2012, 206, p 50-56

69. T. Sekimoto, K. Kurosaki, H. Muta, and S. Yamanaka, Thermoelectric Properties of (Ti, Zr, Hf) CoSb Type Half-Heusler Compounds, Mater. Trans., 2005, 46(7), p 1481-1484

70. G.V. Chester and A. Thellung, The Law of Wiedemann and Franz, Proc. Phys. Soc., 1961, 77(5), p 1005

71. H.-S. Kim, Z.M. Gibbs, Y. Tang, H. Wang, and G.J. Snyder, Characterization of Lorenz Number with Seebeck Coefficient Measurement, APL Mater., 2015, 3(4), p 41506

72. A. Jain and A.J.H. McGaughey, Thermal Conductivity of Compound Semiconductors: Interplay of Mass Density and Acoustic-Optical Phonon Frequency Gap, J. Appl. Phys., 2014, 116(7), p 73503

73. D.P. Spitzer, Lattice Thermal Conductivity of Semiconductors: A Chemical Bond Approach, J. Phys. Chem. Solids, 1970, 31(1), p 19-40

74. C.S. Birkel, J.E. Douglas, B.R. Lettiere, G. Seward, N. Verma, Y. Zhang, T.M. Pollock, R. Seshadri, and G.D. Stucky, Improving the Thermoelectric Properties of Half-Heusler TiNiSn through Inclusion of a Second Full-Heusler Phase: Microwave Preparation and Spark Plasma Sintering of $\mathrm{TiNi}_{1+\mathrm{x}} \mathrm{Sn}$, Phys. Chem. Chem. Phys., 2013, 15(18), p 6990-6997

75. S. Kasap, C. Koughia, H. Ruda, and R. Johanson, Electrical Conduction in Metals and Semiconductors, Springer Handbook of Electronic and Photonic Materials, S. Kasap and P. Capper, Ed., Springer, Cham, 2007, p 19-45

76. H. Lee, D. Vashaee, D.Z. Wang, M.S. Dresselhaus, Z.F. Ren, and G. Chen, Effects of Nanoscale Porosity on Thermoelectric Properties of SiGe, J. Appl. Phys., 2010, 107(9), p 94308

77. K. Valalaki, P. Benech, and A.G. Nassiopoulou, High Seebeck Coefficient of Porous Silicon: Study of the Porosity Dependence, Nanoscale Res. Lett., 2016, 11(1), p 201

78. G.L. Eesley, Relaxation Time of Electrons, McGraw-Hill Education, New York, 2019

79. K. Kirievsky, Y. Gelbstein, and D. Fuks, Phase Separation and Antisite Defects in the Thermoelectric TiNiSn Half-Heusler Alloys, J. Solid State Chem., 2013, 203, p 247-254

80. V.A. Romaka, P. Rogl, V.V. Romaka, E.K. Hlil, Y.V. Stadnyk, and S.M. Budgerak, Features of a Priori Heavy Doping of the N-TiNiSn Intermetallic Semiconductor, Semiconductors, 2011, 45(7), p 850856

81. A. Berche and P. Jund, Oxidation of Half-Heusler NiTiSn Materials: Implications for Thermoelectric Applications, Intermetallics, 2018, 92, p 62-71

82. M. Zou, J.-F. Li, B. Du, D. Liu, and T. Kita, Fabrication and Thermoelectric Properties of Fine-Grained TiNiSn Compounds, J. Solid State Chem., 2009, 182(11), p 3138-3142

83. S.-W. Kim, Y. Kimura, and Y. Mishima, High Temperature Thermoelectric Properties of TiNiSn-Based Half-Heusler Compounds, Intermetallics, 2007, 15(3), p 349-356

84. C.S. Birkel, J.E. Douglas, B.R. Lettiere, G. Seward, Y. Zhang, T.M. Pollock, R. Seshadri, and G.D. Stucky, Influence of Ni Nanoparticle Addition and Spark Plasma Sintering on the TiNiSn-Ni System: Structure, Microstruct. Thermoelectr. Prop. Solid State Sci., 2013, 26, p 16-22

85. J.-L. Chen, C. Liu, L. Miao, J. Gao, Y. Zheng, X. Wang, J. Lu, and M. Shu, Improved Thermoelectric Performance Achieved by Regulating Heterogeneous Phase in Half-Heusler TiNiSn-Based Materials, J. Electron. Mater., 2018, 47(6), p 3248-3253

86. P. Villars and K. Cenzual, Eds., TiNiSn Crystal Structure: Datasheet from 'PAULING FILE Multinaries Edition-2012' in Springer Materials (h ttps://materials.springer.com/isp/crystallographic/docs/sd_1010501), (Springer, Berlin and Material Phases Data System (MPDS), Switzerland \& National Institute for Materials Science (NIMS), Japan, n.d.). https:// materials.springer.com/isp/crystallographic/docs/sd 1010501

87. Y.W. Chai and Y. Kimura, Microstructure Evolution of Nanoprecipitates in Half-Heusler TiNiSn Alloys, Acta Mater., 2013, 61(18), p 6684-6697

Publisher's Note Springer Nature remains neutral with regard to jurisdictional claims in published maps and institutional affiliations. 